\title{
PHF6 and JAK3 mutations cooperate to drive T-cell acute lymphoblastic leukemia progression
}

\author{
Shengnan Yuan $\mathbb{D}^{1,3}$, Xiaomin Wang $\mathbb{D}^{1,2,3 凶}{ }^{凶}$, Shuaibing Hou ${ }^{1}$, Tengxiao Guo ${ }^{1}$, Yanjie Lan ${ }^{1}$, Shuang Yang ${ }^{1}$, Fei Zhao ${ }^{1}$, Juan Gao ${ }^{1}$,

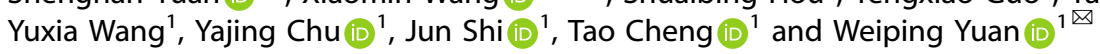

(c) The Author(s) 2021

T-cell acute lymphoblastic leukemia (T-ALL) is a malignant hematologic disease caused by gene mutations in T-cell progenitors. As an important epigenetic regulator, PHF6 mutations frequently coexist with JAK3 mutations in T-ALL patients. However, the role(s) of PHF6 mutations in JAK3-driven leukemia remain unclear. Here, the cooperation between JAK3 activation and PHF6 inactivation is examined in leukemia patients and in mice models. We found that the average survival time is shorter in patients with JAK/STAT and PHF6 comutation than that in other patients, suggesting a potential role of PHF6 in leukemia progression. We subsequently found that Phf6 deficiency promotes JAK3 ${ }^{M 5111}$-induced T-ALL progression in mice by inhibiting the Bai1-Mdm2-P53 signaling pathway, which is independent of the JAK3/STAT5 signaling pathway. Furthermore, combination therapy with a JAK3 inhibitor (tofacitinib) and a MDM2 inhibitor (idasanutlin) reduces the Phf6 KO and JAK3 ${ }^{M 5111}$ leukemia burden in vivo. Taken together, our study suggests that combined treatment with JAK3 and MDM2 inhibitors may potentially increase the drug benefit for T-ALL patients with PHF6 and JAK3 comutation.

Leukemia (2022) 36:370-382; https://doi.org/10.1038/s41375-021-01392-1

\section{INTRODUCTION}

T-cell acute lymphoblastic leukemia (T-ALL) is one of the most common hematologic malignancies, resulted from gene mutations and genomic rearrangements in T-cell progenitors [1, 2]. The 5-year survival rate of adult T-ALL patients is less than $50 \%$, and the mortality rate of relapsed adult T-ALL can be as high as $90 \%$ [3]. Recent studies have shown that T-ALL results from multistep transformation processes that involve the accumulation of genetic defects, including activating mutations of NOTCH1 or JAK-STAT, super-enhancer generating mutations of TAL1, deep deletion of cell cycle-related genes (such as CDKN2, RB1, and CDKN1B), and inactivating mutations of WT1, LEF1, GATA3, and PHF6 [4-6]. The close interrelationship between key regulators of early T-cell development and T-ALL oncogenic signals is best illustrated by the prominent roles of the JAK-STAT signal pathway in T-ALL $[5,7,8]$. More than $25 \%$ of T-ALL patients carry JAK-STAT mutations [9-11], of which JAK3 mutation is the most frequent in T-ALL cases $[8,9,12,13]$. The continuous activation of JAK-STAT signaling has been shown to play an essential role in T-ALL initiation and progression [7, 8, 12, 13].

JAK family kinases are nonreceptor tyrosine kinases that function as signal transducers to activate STAT protein to support the differentiation, proliferation and survival of early T-cell progenitors [10]. Activated STAT proteins translocate to nucleus and act as transcription factors to regulate gene expression and/or induce novel epigenetic changes [11]. Aberrant JAK signaling has been linked with T-ALL development. JAK1 mutations can be found in $10-15 \%$ of T-ALL and $1-2 \%$ of acute myeloid leukemia (AML) patients $[14,15]$. JAK2 $2^{\mathrm{V} 617 F}$ mutation is mainly associated with myeloproliferative neoplasms [16]. JAK3 mutations can be identified in $16.1 \%$ of T-ALL cases. JAK3 plays a key role in regulating $T$ and $B$ cell development $[9,13,17]$, while the rate of the most common JAK3 mutation $(\mathrm{M} 511 \mathrm{l})$ is $34.7 \%$ in all JAK3 mutations $[8,9,13]$. Transplantation of mice with bone marrow (BM) progenitor cells expressing the active $J A K 3^{M 5111}$ mutant allele induces a lymphoproliferative disorder followed by a T-ALL-like disease [12]. Moreover, the JAK3 ${ }^{M 5111}$ mutation induces phosphorylation and activation of STAT5, which subsequently activates the oncogenes to drive leukemia cell proliferation [8, 12].

It has been reported that JAK3 mutations could act as "driver" mutations in T-ALL. Interestingly, JAK3 mutations are frequently accompanied by a high number of genetic changes in T-ALL, such as changes in epigenetic regulatory genes (ASXL1, DNMT3A, EED, EZH2, PHF6, and SUZ12) $[5,13,17,18]$. PHF6 is one of the most common mutated epigenetic regulatory gene coexisting with JAK3 in T-ALL patients $[8,17,19]$. Studies have found that Phf6deficient HSCs had higher proliferation and reconstitution capacity than the wild-type HSCs, although loss of Phf6 alone is not sufficient to induce aberrant hematopoietic transformation [20-23].

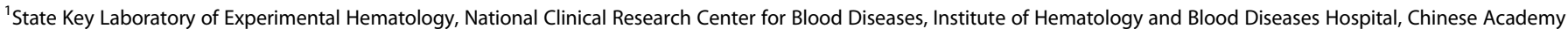

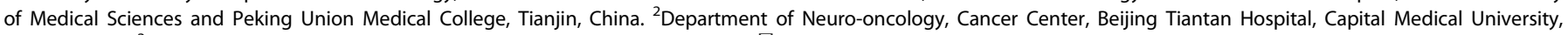
Beijing, China. ${ }^{3}$ These authors contributed equally: Shengnan Yuan, Xiaomin Wang. ${ }^{凶}$ email: wangxiaomin@ihcams.ac.cn; wpyuan@ihcams.ac.cn
} 
Nevertheless, Phf6 loss could significantly accelerate leukemia development driven by aberrant expression of $T L X 3$ or lower the threshold of NOTCH1-induced T-ALL development. Phf6 deficiency could also activate leukemia stem cell transcriptional programs and enhance T-ALL leukemia-initiating cell (LIC) activity [20-22]. While these studies provide mechanistic insight into the importance of PHF6 in regulating early T-cell development, the mechanism of how PHF6 and JAK3 co-occurring events drive leukemogenesis still needs to be functionally dissected and elucidated.

Here we examined the role of cooperatively PHF6 mutations in the context of JAK/STAT mutant-induced leukemia in patients and further functionally dissect their biological events using $J A K 3^{M 5111}$ T-ALL mouse model. We found that PHF6 mutations frequently coexist with JAK3 mutations in T-ALL patients, and Phf6 comutation with $J A K 3$ can drive aggressive leukemia in mice.

\section{MATERIALS AND METHODS}

\section{Generation of leukemia mice models}

Phf6 conditional deletion mice were generated using the homologous recombination technique and mated with Vav1-Cre or Mx1-Cre mice to delete Phf6 in hematopoietic cells. The mice, including donors and recipients used in all our experiments were male mice of 8 weeks. The MSCV-JAK3 ${ }^{\text {M5111 }}$-IRES-GFP retroviral vector was kindly provided by Dr. Jan Cools $[8,12]$. Lineage-negative $\left(\mathrm{Lin}^{-}\right)$cells were transfected with JAK3 $3^{\mathrm{M} 5111}$ GFP virus and transplanted into male mice to establish Vav1-Cre;Phf6 ${ }^{f / y}+$ $J A K 3^{M 5111}$ and $M \times 1-C r e ; P h f 6^{f / y}+J A K 3^{M 5111}$ mice models. Detailed mouse lines used in this study have been listed in supplementary Table 1 (Table S1). This research was approved by the Institute Ethics Committee and the Institutional Animal Care and Use Committee (IACUC), Institute of Hematology and Blood Diseases Hospital, CAMS/PUMC, KT2020052-EC-1.

\section{Extreme limiting dilution analysis of LICs}

The recipient mice were sublethally irradiated and received 500,000, $50,000,5000,500$, or $100 \mathrm{GFP}^{+}$BM cells. The mice were monitored for 4 months. The extreme limiting dilution LIC data were analyzed with ELDA software online [24].

\section{Inhibitor experiments}

For the in vivo experiment, mice were treated through oral gavage with tofacitinib ( $40 \mathrm{mg} / \mathrm{kg}$ per day), idasanutlin $(30 \mathrm{mg} / \mathrm{kg}$ per day) (MedChemExpress, New Jersey, USA), or vehicle ( $0.2 \%$ dimethylsulfoxide [DMSO]). The mice received medication daily until death. For the in vitro experiment, MOLT-4 cells were treated with tofacitinib $(2.0 \mu \mathrm{M})$, idasanutlin $(0.5 \mu \mathrm{M})$ or $0.2 \%$ DMSO in culture medium for $48 \mathrm{~h}$ and then proliferation and apoptosis were examined.

\section{RNA-Seq and ChIP-Seq assay}

For RNA-Seq analysis of Phf6 WT/KO + JAK3 ${ }^{M 5111}$ cells, $1 \mu \mathrm{g}$ RNA per sample was used for RNA sample preparations. Transcriptome sequencing was performed on Illumina NovaSeq 6000 platform (Illumina, CA, USA) to a total target depth of 10 million 150 bp paired end reads. Differential expression analysis was performed by DESeq2 $\mathrm{R}$ package (1.16.1).

The ChIP assay was performed with the ChIP assay kit (Cell Signaling Technology, Boston, MA, USA) according to manufacturer's recommendations. Chromatin from cross-linked HA-PHF6-overexpressed K562 or control cells was sheared using an ultrasonicator (Covaris, S220, ABI, New York, USA) to obtain DNA (100-400 bp). Immunoprecipitation was conducted with ChIP-grade HA-tag antibody (Abcam, ab9110, Cambridge, UK), PHF6 antibody (Sigma-Aldrich, HPA001023, Missouri, USA) or normal IgG antibody (Cell Signaling Technology, CST2729, Boston, MA, USA).

RNA-Seq and ChIP-Seq data are available at GEO under accession number GSE159444 and GSE159549.

\section{RESULTS \\ PHF6 mutation frequently co-exists with JAK3 mutation in acute leukemia patients}

We analyzed the genetic data of 449 T-ALL cases from different clinical centers, among which JAK/STAT mutations occurred in
98 samples $(21.83 \%)[13,17,25]$. JAK/STAT and PHF6 comutation accounted for $7.80 \%$ (35/449) of all T-ALL cases. Interestingly, JAK3 and PHF6 comutation accounted for $4.90 \%$ of the 449 cases (Fig. 1A, B). PHF6 mutation occurred in $44.90 \%$ of patients with JAK3 mutation $(P<0.001$, Fig. $1 C$, left). JAK3 mutation occurred in $25.00 \%$ of PHF6-mutated patients and was significantly associated with PHF6 mutation ( $P<0.001$, Fig. $1 C$, right).

We analyzed PHF6 and JAK/STAT mutations in $44 \mathrm{~T} /$ myeloid mixed phenotype acute leukemia cases (T/M MPAL) from 102 MPAL patients in Alexander et al. [19]. We found that PHF6 and JAK/STAT comutation accounted for $6.82 \%(3 / 44)$ in total cases (Fig. 1D, E and Table S2). We assigned the $44 \mathrm{~T} /$ myeloid MPAL patients into four groups including PHF6 and JAK/STAT comutation, single JAK/STAT or PHF6 mutation, and non-PHF6/JAK/ STAT mutation (others), and found that the survival time of the PHF6 and JAK/STAT comutation group was significantly shorter than the single JAK/STAT mutation group $(P<0.0001)$ or the non-PHF6/JAK/STAT mutation group $(P<0.0001$, Figs. $1 \mathrm{E}$ and $\mathrm{S} 1 \mathrm{~A})$. The combined clinical data suggested that PHF6 mutations may play a synergetic role with JAK/STAT mutations in leukemia development.

\section{PHF6 mutation acts as an early event to accelerate JAK3 ${ }^{M 511 I}$} hematopoietic progenitor cell transformation

To evaluate the potential role of PHF6 mutation in leukemiainitiating events, we generated Phf6 knockout (Vav1-Cre;Phf6 ${ }^{f / y}$ ) and wild-type $\left(P h f \sigma^{f / y}\right)$ mice. We sorted Lin $^{-}$cells from BM of Vav1Cre;Phf6 ${ }^{f / y}$ or Phf6 ${ }^{f / y}$ male mice and transfected with JAK3 ${ }^{M 5111}$-GFP virus. Equal number of $\mathrm{GFP}^{+}$cells were transplanted into male recipients to establish Vav1-Cre;Phf6 ${ }^{f / y}+J A K 3^{M 5111}$ (presented as VC Phf6 $+J A K 3^{M 5111}$ ) and Phf6 ${ }^{f / y}+J A K 3^{M 5111}$ (as Phf6 WT + $J A K 3^{M 5111}$ ) mouse lines (Figs. $2 A$ and S1B) $[8,12]$. While all mice succumbed to leukemia, the survival time of VC Phf6 $+J A K 3^{M 5111}$ mice was significantly shorter than Phf6 WT $+J A K 3^{M 5111}$ mice (Fig. 2B). The percentage of $\mathrm{GFP}^{+}$leukemia cells was higher in the peripheral blood (PB) of VC Phf6 $+J A K 3^{M 5111}$ mice than Phf6 WT + JAK3 ${ }^{M 5111}$ mice (Figs. $2 \mathrm{C}$ and S1C). Although $\mathrm{GFP}^{+}$leukemia cells in the two groups were mainly $\mathrm{CD}^{+}$or $\mathrm{CD}^{+}{ }^{+}$, the percentages of myeloid and $B$ cells were significantly increased in the PB of VC Phf6 $+J A K 3^{M 5111}$ mice when compared with Phf6 WT $+J A K 3^{M 5111}$ mice (Fig. S1D, E). The VC Phf6 $+J A K 3^{M 5111}$ mice showed more aggressive leukemia phenotypes than Phf6 WT $+J A K 3^{M 5111}$ mice, including higher white blood cells (WBCs), neutrophils (Neu), and lymphocytes (Lym) in PB (Figs. 2D, E and S1F). The weights of spleen, liver, and thymus were slightly increased in VC Phf6 + $J A K 3^{M 5111}$ mice when compared with Phf6 WT + JAK3 ${ }^{M 5111}$ mice (Fig. S1G-J). The percentage of $\mathrm{GFP}^{+}$leukemia cells and the degree of extramedullary infiltration in spleen, liver, lung, and brain, were increased in VC Phf6 $+J A K 3^{M 511 I}$ mice than Phf6 WT + $J A K 3^{M 5111}$ mice, (Fig. 2F, G). These results demonstrated that Phf6 deficiency accelerated the initiation of JAK3 $3^{M 511}$ hematopoietic progenitor cell transformation.

To further investigate the role of Phf6 deletion in the transformation of hematopoietic progenitors with JAK3 mutations, we quantified the biological characteristics of VC Phf6 $+J A K 3^{M 5111}$ cells in BM with various surface markers. The percentage of $\mathrm{GFP}^{+}$ cells was higher in BM of VC Phf6 $+J A K 3^{M 511 l}$ mice (44.1\%) than the Phf6 WT $+J A K 3^{M 5111}$ mice $(7.8 \%)$ (Fig. $\left.2 \mathrm{H}, \mathrm{I}\right)$. The percentages of myeloid and B cells in the BM of VC Phf6 $+J A K 3^{M 5111}$ mice were $20.9 \%$ and $25.7 \%$ respectively, and were higher than the controls (Fig. $2 \mathrm{l}, \mathrm{J})$. Wright-Giemsa staining revealed the development of a complex hematolymphoid neoplasm characterized by the coexistence of different populations of atypical cells displaying both lymphoid and myeloid differentiation in VC Phf6 $+J A K 3^{M 5111}$ mice when compared to the controls (Fig. 2K). These results suggested that Phf6 deficiency induced the polyclonal expansion of JAK3 ${ }^{M 5111}$ hematopoietic progenitors. 
A

\begin{tabular}{lllccc}
\hline & Liu et al, 2017 & Vicente et al, 2015 & Spinella et al, 2016 & Total $(\mathrm{n}=449)$ & Percent \% \\
\hline JAK/STAT & $42 / 264(15.91 \%)$ & $51 / 155(32.90 \%)$ & $5 / 30(16.67 \%)$ & 98 & 21.83 \\
JAK3 & $20 / 264(7.58 \%)$ & $25 / 155(16.13 \%)$ & $4 / 30(13.33 \%)$ & 49 & 10.91 \\
PHF6 & $50 / 264(18.94 \%)$ & $30 / 155(19.35 \%)$ & $8 / 30(26.67 \%)$ & 88 & 19.60 \\
JAK/STAT+PHF6 & $16 / 264(6.06 \%)$ & $16 / 155(10.32 \%)$ & $3 / 30(10.00 \%)$ & 35 & 7.80 \\
JAK3+PHF6 & $7 / 264(2.65 \%)$ & $12 / 155(7.74 \%)$ & $3 / 30(10.00 \%)$ & 22 & 4.90 \\
\hline
\end{tabular}

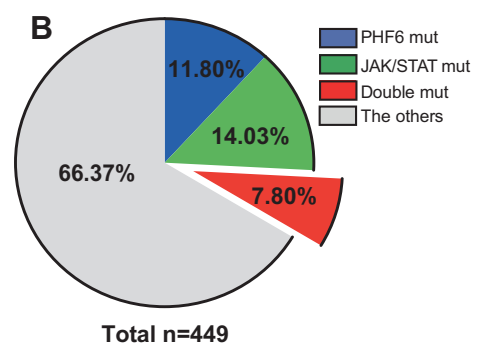

C

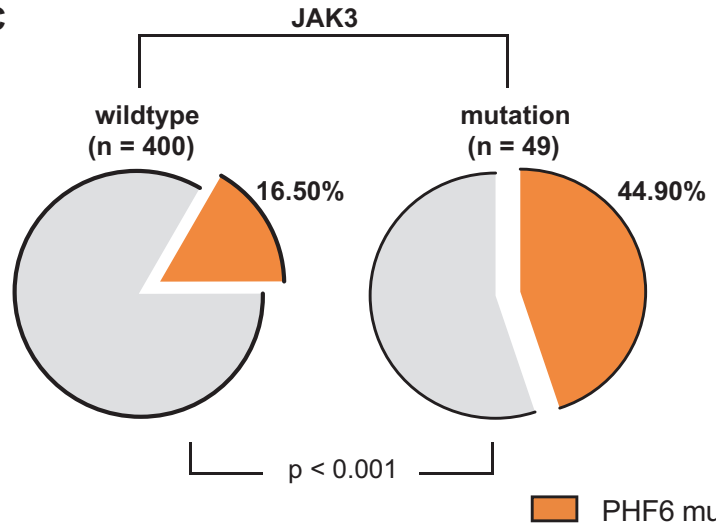

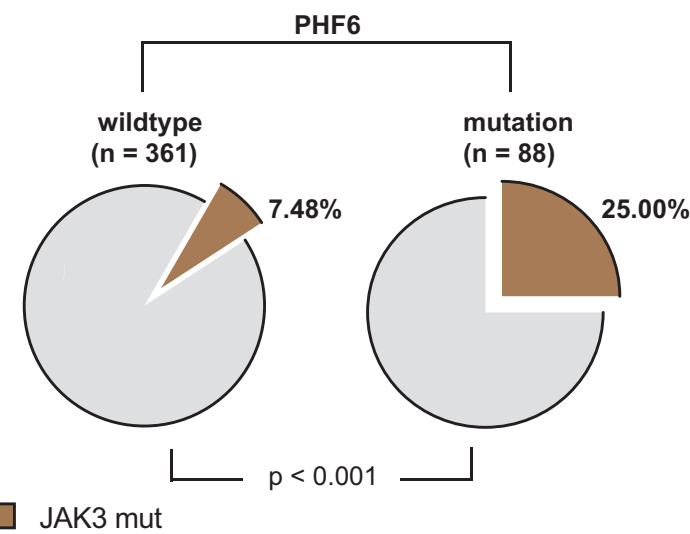

JAK3 mut

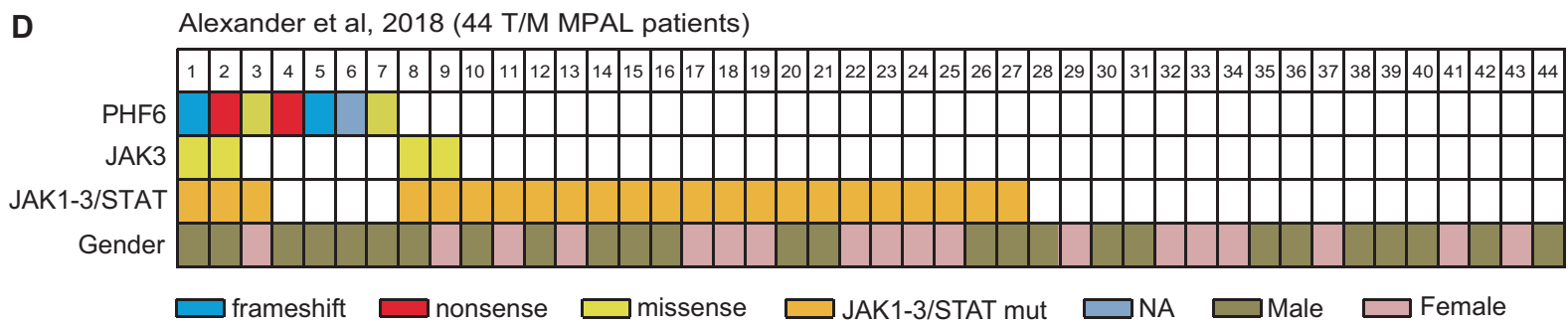

E

\begin{tabular}{|c|c|c|c|c|c|c|}
\hline Mutation & $\begin{array}{l}\text { T/M patients } \\
\quad(\mathrm{n}, \%)\end{array}$ & & $\begin{array}{c}\text { Median age } \\
\text { years (range) }\end{array}$ & $\begin{array}{l}\text { Male: } \\
\text { Female }\end{array}$ & $\begin{array}{l}\text { Mutation } \\
\text { type }\end{array}$ & $\begin{array}{l}\text { Average survival } \\
\text { time (Days) }\end{array}$ \\
\hline Single PHF6 mut & $4(9.09)]$ & \multirow{3}{*}{$\begin{array}{l}7(15.91) \\
23(52.27)\end{array}$} & $6.5(5 \sim 9)$ & $4: 0$ & fs, non, mis & 854 \\
\hline Single JAK/STAT mut & $20(45.45)$ & & $12(2 \sim 17)$ & $10: 10$ & mainly missense & 1483 \\
\hline $\begin{array}{c}\text { JAK/STAT+PHF6 } \\
\text { co-mut }\end{array}$ & 3 ] ل 3 (6.82) & & $9(6 \sim 10)$ & $2: 1$ & $\begin{array}{l}\text { PHF6: fs, non, mis } \\
2 \text { JAK3: M511I (mis) } \\
\text { 1 STAT5B (mis) }\end{array}$ & 102 \\
\hline The others & $17(38.64)$ & & $4(1 \sim 16)$ & $10: 7$ & I & 1423 \\
\hline
\end{tabular}

Fig. 1 PHF6 mutations are associated with JAK3 mutations in AL patients. A, B The percentage of JAK3 or PHF6 mutations in 449 T-ALL patients from three independent clinical centers. C Left panel, the frequency of PHF6 mutations (orange) in T-ALL patients with JAK3 mutations vs the frequency of PHF6 mutations in T-ALL patients with WT JAK3. Right panel, the frequency of JAK3 mutations (orange) in T-ALL patients with PHF6 mutations vs the frequency of JAK3 mutations in T-ALL patients with WT PHF6. Fisher's exact test was applied to calculate the $P$ values to analyze the significance of the positive association between JAK3 mutations and PHF6 mutations. D Frameshift (fs), nonsense (non), and missense (mis) of PHF6 or JAK3 found in 44 T/M MPAL cases from Alexander et al. [19]. Each type of mutation is indicated by a unique color. Gender are also shown for each patient. $\mathbf{E}$ Total case number, median age, gender ratio, mutation type and average survival time of 44 T/M MPAL patients from Alexander et al. [19].

Phf6 deletion promotes JAK3 ${ }^{M 511}{ }_{\text {-induced T-ALL progression }}$ Since JAK3 mutations could act as "driver" mutations in leukemia patients, we investigated whether PHF6 mutations act as subsequent events to promote $J A K 3^{M 5111}$-induced leukemia development by generating Mx1-Cre;Phf6 ${ }^{f / y}\left(M C P h f 6^{f / y}\right)$ and
Mx1-Cre;Phf6 ${ }^{+/ y}(M C)$ mice, and constructed MCPhf6 ${ }^{f l y}+J A K 3^{M 5111}$ and $M C+J A K 3^{M 5111}$ mice (Fig. 3A). MC Phff fl/y or MC Lin $^{-}$cells were transfected with retrovirus containing JAK3 ${ }^{M 5111} /$ GFP ex vivo, and equal number of $\mathrm{GFP}^{+}$cells were injected into lethally irradiated mice. plpC was injected at 3 weeks post-transplantation 


\section{A}

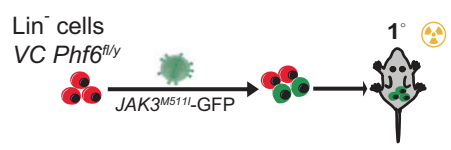

Lin cells
Phf6
B $\quad$ - Phf6 WT+JAK3 ${ }^{M 5111} \mathrm{n}=15$

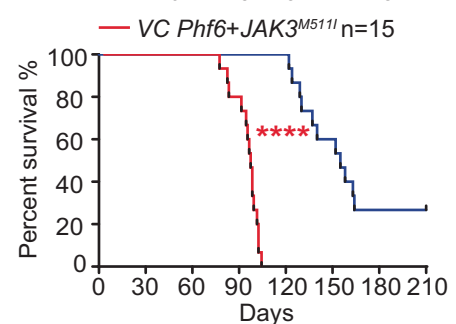

E

D Phf6 WT+JAK3 $3^{M 111}$

- VC Phf6+JAK3 ${ }^{M 511}$

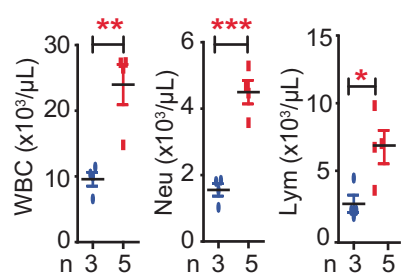

$\mathrm{PB}$
C $\quad$ - Phf6 WT+JAK3 ${ }^{M 5111}$

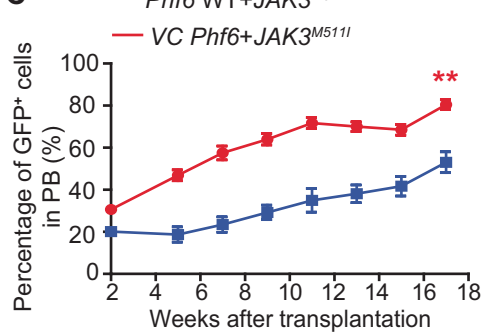

F - Phf6 WT+JAK3 $3^{M 111}$

- VCPhf6+JAK3 ${ }^{M 511}$

@ Spleen $\widehat{\circ}$ Liver $\cong$ Thymus

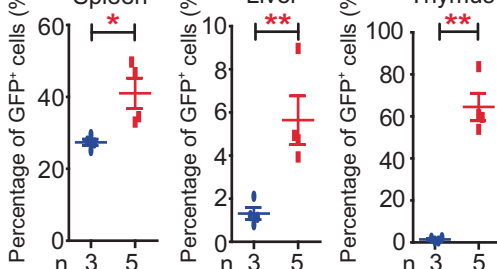

G
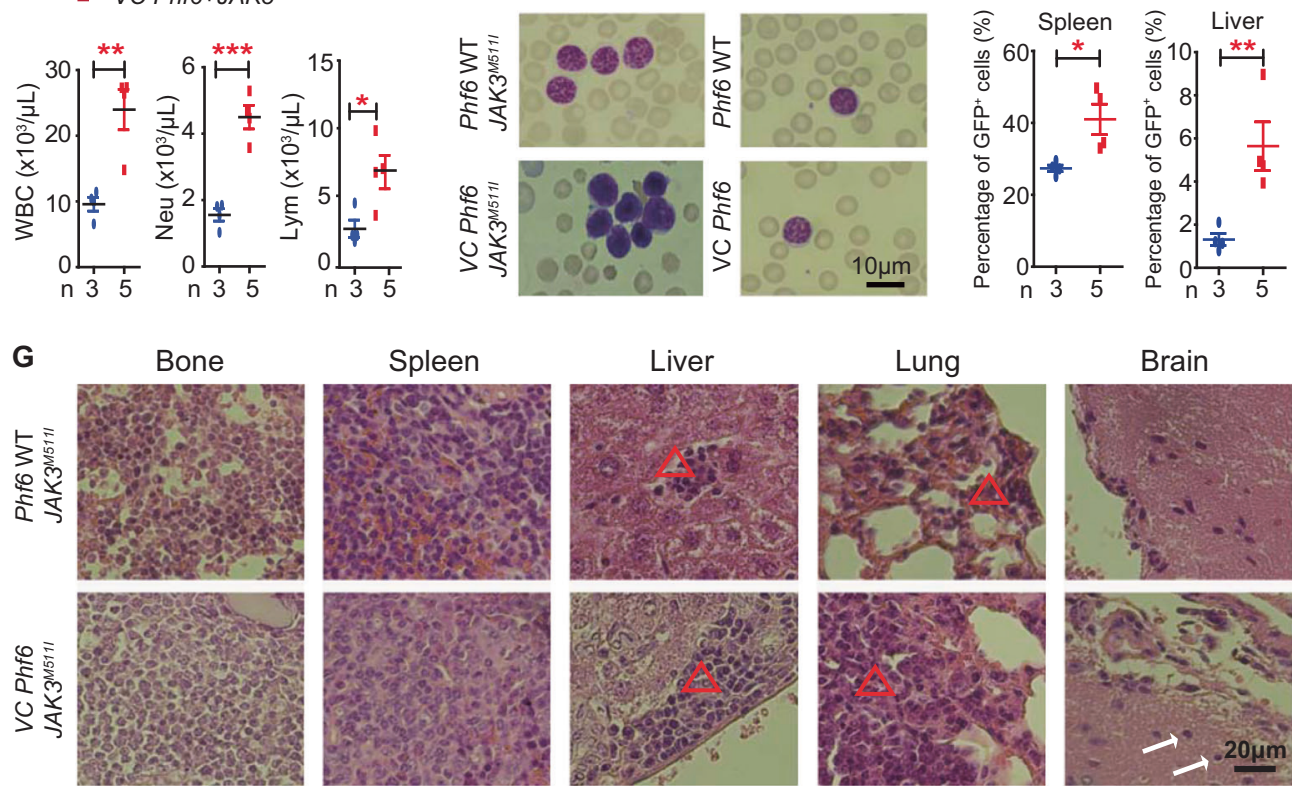

H

I

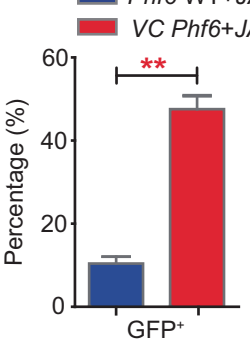

J

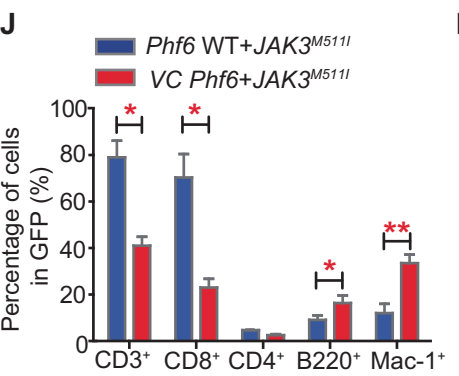

K

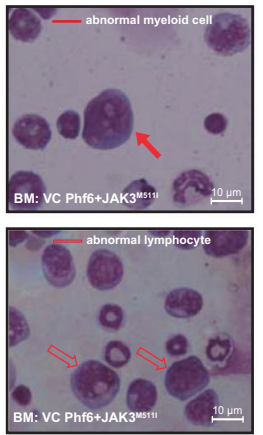

$\mathrm{GFP}^{+}$

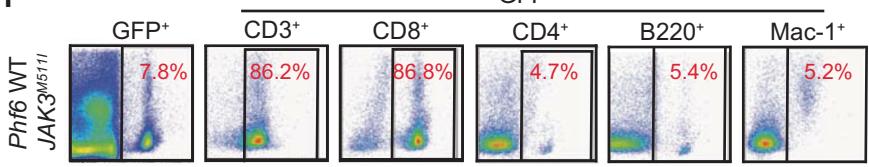

낭ำ
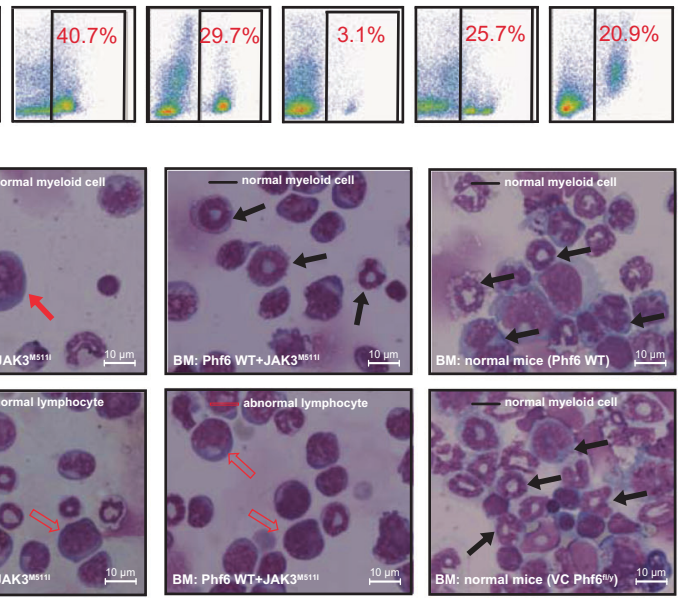

to delete Phf6 when $\mathrm{CD}^{+}$cells in $\mathrm{GFP}^{+}$cells were above $70 \%$ in $J A K 3^{M 5111}$-induced T-cell leukemia (Fig. 3A, B). Phf6 deletion was confirmed by western blotting of BM cells from MC Phff ${ }^{f l y}+$ $J A K 3^{M 5111}$ and $M C+J A K 3^{M 5111}$ mice treated with plpC (presented as MC Phf6 KO $+J A K 3^{M 5111}$ or MC Phf6 WT $+J A K 3^{M 5111}$ ) (Fig. 3C). We sorted $\mathrm{GFP}^{+}$cells from BM of MC Phf6 WT $+J A K 3^{M 5111}$ and MC Phf6 $\mathrm{KO}+J A K 3^{M 5111}$ mice, and performed Wright-Giemsa staining to discern the morphological characteristics of $\mathrm{GFP}^{+}$cells. We found that most of the GFP ${ }^{+}$cells in BM of the two mouse groups were abnormal lymphocytes with larger cell size, irregular nuclear 
Fig. 2 Phf6 deletion and JAK3 ${ }^{M 5111}$ overexpression lead to rapid hematopoietic progenitor transformation to leukemia cells. A Scheme of constructing VC Phf6 +JAK3 ${ }^{M 5111}$ and Phf6 WT $+J A K 3^{M 5111}$ T-ALL mouse models. B Kaplan-Meier survival curves of VC Phf6 + JAK3 ${ }^{M 5111}$ T-ALL mice and Phf6 WT $+J A K 3^{M 5111}$ T-ALL mice (log-rank test $P<0.0001, n=15$ per group). VC Phf6 $+J A K 3^{M 5111}$ T-ALL mice (median survival time $=$ 85 days), Phf6 WT + JAK3 ${ }^{M 5111}$ T-ALL mice (median survival time $=143$ days). C Percentage of GFP ${ }^{+}$leukemia cells in PB at different time points. D The counts of WBCs, lymphocytes (Lym), and neutrophils (Neu) in PB by routine blood tests. E Wright-Giemsa staining of peripheral blood cells. F Percentage of GFP ${ }^{+}$cells in spleen, liver, and thymus. G Hematoxylin and eosin (HE) staining of BM, spleen, liver, lung, and brain. A red triangle or white arrow indicates the leukemia infiltration area. H Percentage of GFP ${ }^{+}$leukemia cells in BM at 21 weeks after transplantation. I, J Percentages of T cells, B cells, and myeloid cells in the GFP ${ }^{+}$population in BM at 21 weeks after transplantation. $\mathbf{K}$ Wright-Giemsa staining of BM cells.

contours, and prominent nucleoli (Fig. S1K). Interestingly, both $M C$ Phf6 WT $+J A K 3^{M 5111}$ and MC Phf6 KO $+J A K 3^{M 5111}$ mice developed $\mathrm{CD}^{+}{ }^{\mathrm{T}}$-ALL without myeloid expansion (Fig. 3D, E). Furthermore, the percentages of $\mathrm{GFP}^{+}$cells in $\mathrm{PB}, \mathrm{BM}$, spleen, liver, and thymus were higher in MC Phf6 KO + JAK3 ${ }^{M 5111}$ than in MC Phf6 WT + $J A K 3^{M 5111}$ mice (Fig. 3F, G). The WBC and lymphocyte counts were also increased, while the platelet count was decreased in the PB of $M C$ Phf6 KO $+J A K 3^{M 5111}$ mice (Fig. $\left.3 \mathrm{H}\right)$. The weights of spleens and livers of MC Phf6 KO $+J A K 3^{M 5111}$ were much higher than MC Phf6 $\mathrm{WT}+J A K 3^{M 5111}$ mice (Fig. 3l). Importantly, the survival time of $M C$ Phf6 KO + JAK3 ${ }^{M 5111}$ mice was significantly shorter than MC Phf6 WT $+J A K 3^{M 5111}$ mice (Fig. 3J). These data indicated that Phf6 mutation acquired after JAK/STAT mutation could promote $J A K 3^{M 5111}$-induced T-ALL progression.

\section{Phf6 deficiency increases the activity of leukemia-initiating cells in T-ALL}

To determine whether Phf6 deficiency promotes leukemia initiation by increasing LIC number or activity, GFP ${ }^{+}$primary Phf6 WT + JAK3 ${ }^{M 5111}$ and VC Phf6 + JAK3 $3^{M 5111}$ cells were sorted and transplanted into secondary recipients (Fig. 4A). The mice transplanted with VC Phf6 $+J A K 3^{M 5111}$ cells had more leukemic cells in the $\mathrm{PB}, \mathrm{BM}$ and extramedullary organs than the controls (Figs. 4B, C and S2A), with significantly shorter survival time than the controls (Fig. 4D). Importantly, secondary leukemia cells arising from VC Phf6 $+J A K 3^{M 5111}$ primary cells in the BM were mainly lymphoid cells (96.3\%) (Figs. $4 \mathrm{E}$ and S2B), in contrast to the primary VC Phf6 $+J A K 3^{M 5111}$ cells showed much greater heterogeneity (Fig. 2I). Wright-Giemsa staining showed that most GFP ${ }^{+}$ cells of the two groups were abnormal lymphocytes with typical lymphoblastic characteristics (Fig. S2C). These results suggested that the $\mathrm{GFP}^{+}$cells were mainly $\mathrm{T}$ leukemic cells based on immunophenotypes and morphological characteristics and the $\mathrm{GFP}^{+} \mathrm{CD}^{+}$T-cell subclone had a dominant growth advantage in VC Phf6 + JAK3 ${ }^{M 5111}$ cell populations.

To further identify the properties of VC Phf6 $+J A K 3^{M 5111}$ leukemia cells, we analyzed T-cell surface markers of $\mathrm{GFP}^{+}$cells (CD4, CD8, CD25, and CD127). The CD25 marker is highly expressed in T cells during embryonic development [26], and we found that the percentage of $\mathrm{GFP}^{+} \mathrm{CD} 25^{+}$cells was increased in VC Phf6 $+J A K 3^{M 5111}$ mice than the controls, while no obvious CD127 expression was observed (Fig. 4F). Notably, the expression of TCR- $\beta$ was significantly decreased in Phf6-deficient leukemia cells, suggesting that VC Phf6 $+J A K 3^{M 5111}$ cells were more immature (Fig. 4G). Consistent with the presence of more immature T cells in the VC Phf6 $+J A K 3^{M 5111}$ populations, extreme limiting dilution assay demonstrated a marked increase in LICs activity in Phf6 KO T-ALL cells when compared with Phf6 WT T-ALL cells, indicating that loss of Phf6 promoted LICs self-renewal and proliferation (Figs. 4H-J and S2D).

\section{Loss of Phf6 enhances leukemia cell proliferation through acceleration of the cell cycle}

To identify the definitive role of Phf6 deficiency in leukemia cell over-proliferation, we investigated cell cycle and apoptosis in VC Phf6 $+J A K 3^{M 5111}$ cells. We found more VC Phf6 $+J A K 3^{M 5111}$ cells were in $\mathrm{G} 1 / \mathrm{S}$ and $\mathrm{G} 2 / \mathrm{M}$ stages than the control cells (Fig. 5A). However, the cell apoptosis rate was similar in both groups in vivo (Fig. S3A).

To explore the underlying molecular mechanisms of Phf6 loss in accelerating T-ALL cell proliferation, we analyzed the transcriptional profiles of VC Phf6 $+J A K 3^{M 5111}$ and Phf6 WT $+J A K 3^{M 5111}$ T-ALL cells. It revealed a distinct gene expression signature in VC Phf6 $+J A K 3^{M 5111}$ cells (2377 genes upregulated and 3751 genes downregulated; $P<0.05$ ) (Fig. S3B) and these differentially expressed genes were significantly enriched for activities associated with cell cycle, apoptosis, adhesion and lymphocyte differentiation (Fig. 5B). We identified 35 cell cycle-related genes were upregulated, and 39 apoptosis-related genes were downregulated $(P<0.05)$ (Fig. 5C). We further validated that members of $\mathrm{Mcm}$ and $\mathrm{Cdc}$ gene families, considered to enhance cell proliferation, were upregulated, while genes related to apoptosis, such as Caspase family genes, were downregulated in VC Phf6 + JAK3 ${ }^{M 5111}$ cells (Fig. 5D, E). Notably, the analysis showed Phf6 loss inhibited the P53 signaling pathway, as this was validated by the decreased mRNA expression of Gadd45a, Gadd45b and Fasl in Phf6 KO T-ALL cells when compared with the control cells (Fig. 5F).

GO and KEGG analysis revealed active nuclear division and cell cycle in VC Phf6 + JAK3 ${ }^{M 5111}$ cells (Fig. S3C, D), while GSEA showed upregulation of cell cycle progression and DNA replication gene expression in VC Phf6 + JAK3 ${ }^{M 5111}$ cells (Fig. 5G). T-cell maturation might be altered since naive $\mathrm{CD}^{+}$T-cell-related genes were enriched in VC Phf6 $+J A K 3^{M 5111}$ when compared with the controls (Figs. $5 \mathrm{H}$ and $\mathrm{S} 3 \mathrm{E}$ ). Meanwhile, embryonic stem cell related genes were enriched in VC Phf6 $+J A K 3^{M 5111}$ cells (Fig. 5I). These data suggested that Phf6 loss promoted JAK3 ${ }^{M 5111}$ induced T-ALL progression by accelerating the cell cycle and increasing immature T cells.

\section{Phf6 deficiency increases Bai1-mediated P53 degradation}

To determine the underlying molecular mechanism driving the

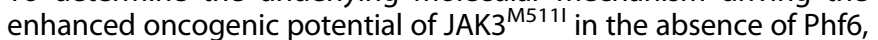
we examined the phosphorylation level of Stat5 in Phf6 WT + $J A K 3^{M 5111}$, VC Phf6 $+J A K 3^{M 5111}$, and WT cells. The p-Stat5 was increased in both Phf6 WT $+J A K 3^{M 5111}$ and VC Phf6 $+J A K 3^{\text {M5111 }}$ when compared with WT cells, while Phf6 loss did not further enhance p-Stat5 in VC Phf6 $+J A K 3^{M 5111}$ when compared with Phf6 WT $+J A K 3^{M 5111}$ cells (Fig. S4A). We further probed that how Phf6 downregulates P53 signaling pathway, and found that Phf6 deficiency decreased P53 protein expression but not P53 mRNA expression in mouse leukemia cells (Fig. 6A left panel, B left panel). To investigate if PHF6 regulates P53 expression independent of the JAK3-STAT5 signaling pathway, we examined P53 protein levels in PHF6 knockdown (KD) U2OS cells treated with X-ray $(35 \mathrm{~Gy})$. We found that the protein expression of P53 was decreased, while the mRNA expression of P53 was unchanged in PHF6 KD U2OS cells when compared with the controls (Fig. 6A right panel, B right panel). The ubiquitination of P53 was significantly increased in PHF6 KD U2OS cells and PHF6 KD MOLT-4 T-ALL cells when compared with the controls (Fig. 6C). These results indicated that PHF6 regulates the ubiquitination of P53. 


\section{A}

$M C+J A K 3^{M 5111}$ cells or MC Phf6 ${ }^{f / y}+J A K 3^{M 5111}$ cells

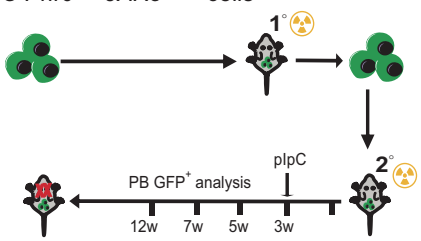

C

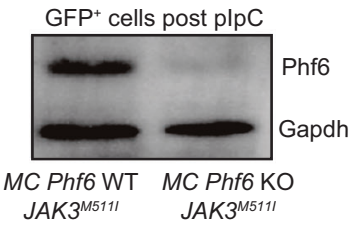

E $\quad M C P h f 6 \mathrm{WT}+J A K 3^{M 5111}$ - MC Phf6 KO+JAK3 ${ }^{\text {M111 }}$

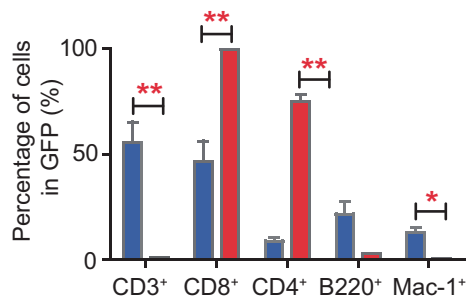

G

- MC Phf6 WT+JAK3 $3^{M 11}$

- MC Phf6 KO+JAK3 ${ }^{M 511}$
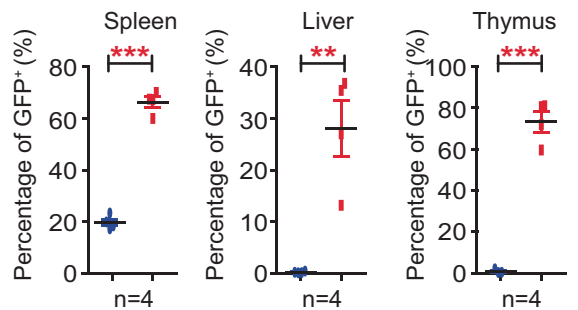

I $-M C$ Phf6 WT+JAK3 ${ }^{M 5111}$

- MC Phf6 KO+JAK3 ${ }^{M 111}$
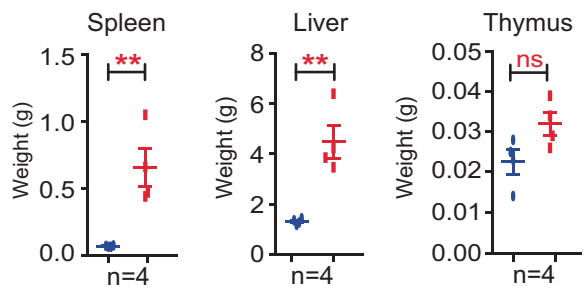

B
MC+JAK3 $3^{M 111}$

$M C P h f^{f l y}+J A K 3^{M 5111}$
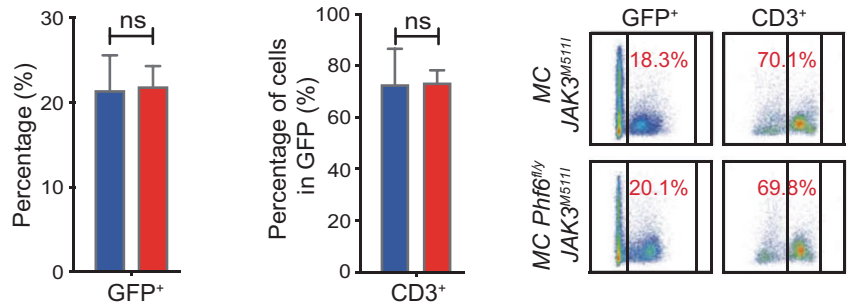

BM GFP $^{+}$

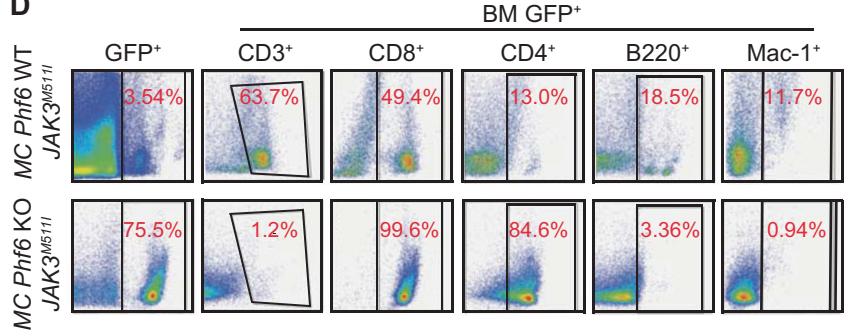

F $\quad M C P h f 6 \mathrm{WT}+J A K 3^{\mathrm{M} 5111}$ - MC Phf6 KO+JAK3 ${ }^{M 5111}$

- MC Phf6 WT+JAK3 $3^{M 111}$ - MC Phf6 KO+JAK3 ${ }^{M 111}$
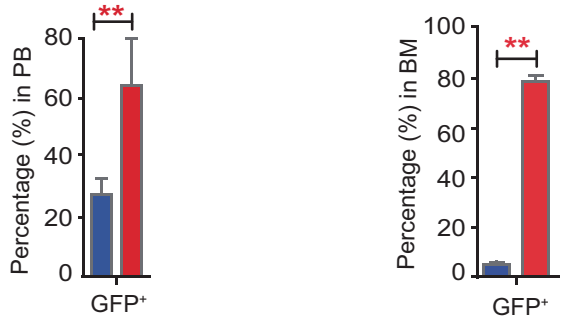

H

- MC Phf6 WT+JAK3 $3^{M 111}$

- MC Phf6 KO+JAK3 ${ }^{M 5111}$
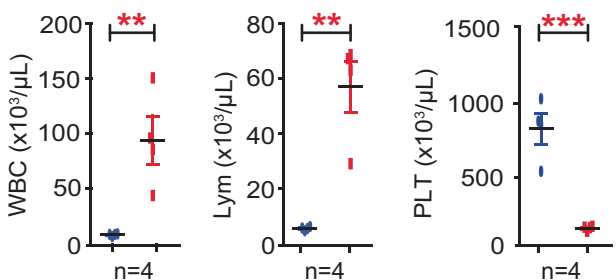

J $\quad M C P h f 6 W T+J A K 3^{M 5111} \mathrm{n}=12$ - MC Phf6 KO+JAK3 $3^{M 111} \mathrm{n}=16$

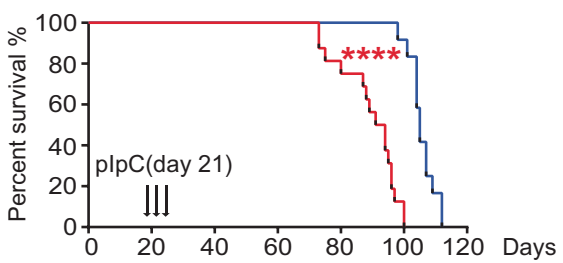

Fig. 3 PHF6 deficiency accelerates T-ALL development in the context of JAK3 mutation. A Schematic representation of Phf6 deletion after $J A K 3^{M 5111}$-induced T-ALL in a mouse model. B Left panel, percentage of GFP ${ }^{+}$leukemia cells in the PB of mice at 3 weeks after transplantation. Right panel, percentage of $\mathrm{CD}^{+}$cells in GFP ${ }^{+}$leukemia cells in the PB of mice at 3 weeks after transplantation. C The Phf6 protein expression level in GFP ${ }^{+}$cells of T-ALL mice treated with plpC. D, E Percentage of T cells, B cells and myeloid cells in GFP ${ }^{+}$cells in the BM of T-ALL mice treated with plpC. F Percentage of GFP ${ }^{+}$cells in the PB and BM of T-ALL mice treated with plpC. G Percentage of GFP ${ }^{+}$cells in the spleen, liver, and thymus of T-ALL mice treated with plpC. $\mathbf{H}$ The counts of WBCs, lymphocytes and platelets in the PB of T-ALL mice treated with plpC by routine blood tests. I The weights of the spleen, liver, and thymus of T-ALL mice treated with plpC. J Kaplan-Meier survival curves of MC Phf6 $\mathrm{KO}+J A K 3^{M 5111} \mathrm{~T}$-ALL mice $(n=16)$ and MC Phf6 WT $+J A K 3^{M 1111}$ T-ALL mice $(n=12)$ after plpC injection (log-rank test $\left.P<0.0001\right)$. 
A

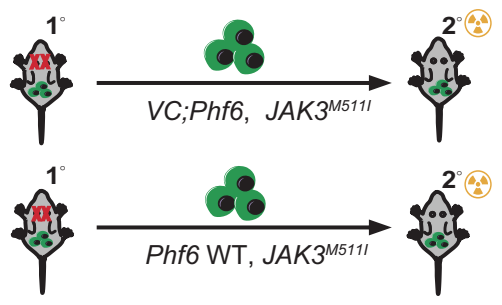

D - Phf6 $\mathrm{WT}+J A K 3^{M 511} \mathrm{n}=10$

- VC Phf6+JAK3 $3^{M 111} \mathrm{n}=10$

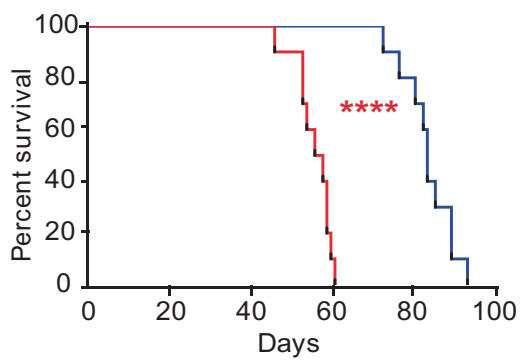

G

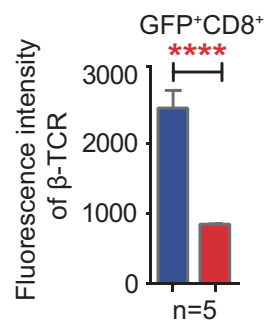

I

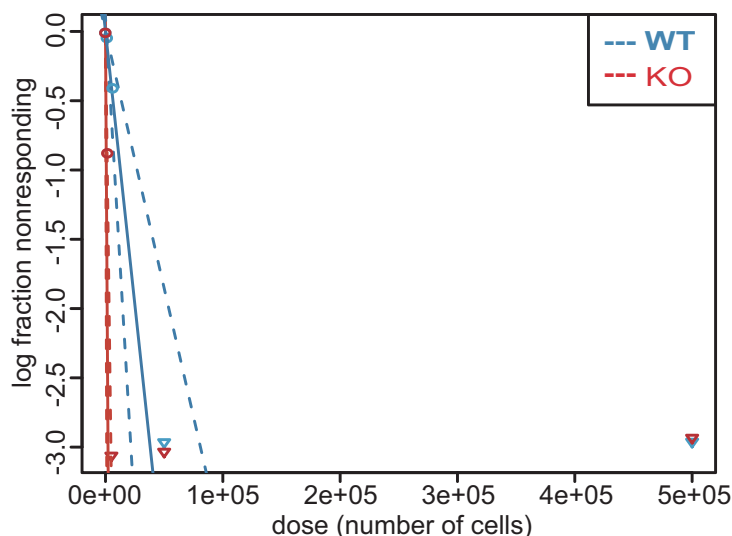

B

Phf6 WT+JAK3 $3^{M 111}$ VC Phf6+JAK3 $3^{M 111}$
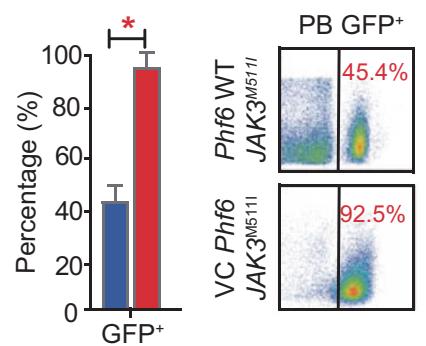

E Phf6 WT+JAK3 ${ }^{\text {M511 }}$

VC Phf6+JAK3 $3^{\text {M111 }}$

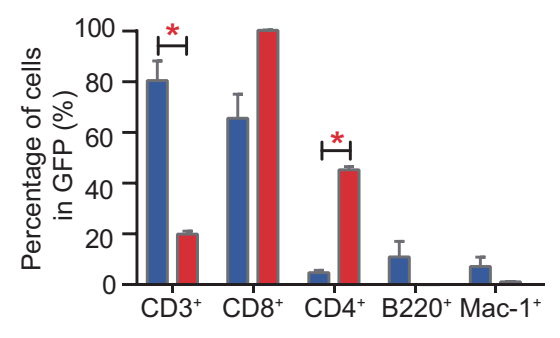

H
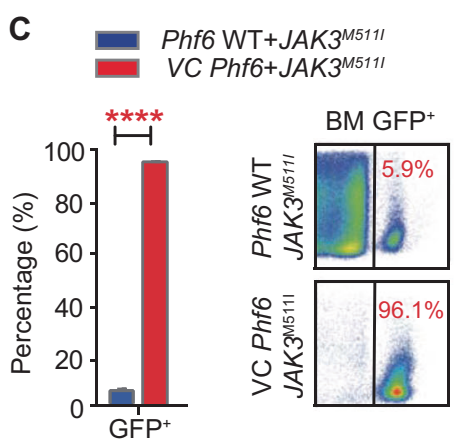
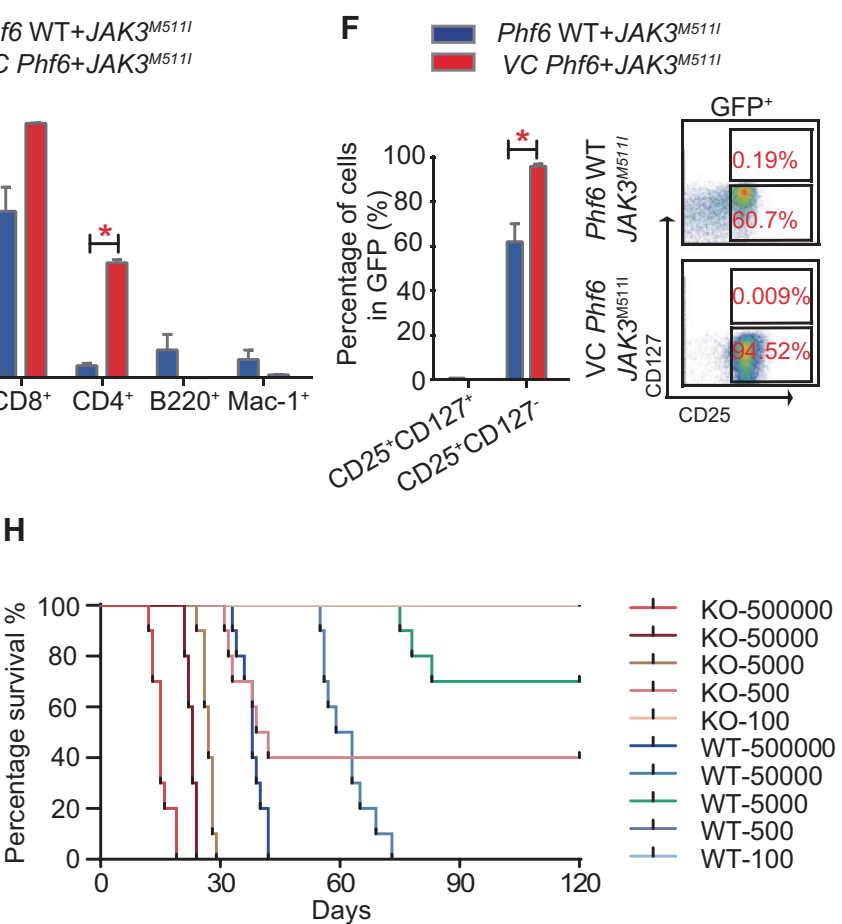

J

Confidence intervals for
1/(stem cell frequency)
\begin{tabular}{|c|c|c|c|}
\hline Group & Lower & Estimate & Upper \\
\hline KO & 1531 & 713 & 332 \\
\hline WT & 26977 & 12694 & 5973 \\
\hline \multicolumn{3}{|c|}{} \\
\hline Chisq & DF & P.value \\
\hline 26.2 & 1 & 3.16 E-07 \\
\hline
\end{tabular}

Fig. 4 Phf6 deletion increases the activity of LICs in T-ALL mice. A Schematic representation of secondary transplantation. B, C Percentage of GFP leukemia cells in the PB and BM of mice at 8 weeks after secondary transplantation. D Kaplan-Meier survival curves of VC Phf6 + JAK3 ${ }^{M 5111}$ T-ALL mice and Phf6 WT $+J A K 3^{M 5111}$ T-ALL mice in the secondary transplantation assay (log-rank test $P<0.0001, n=10$ per group). E Percentage of T cells, B cells and myeloid cells in GFP ${ }^{+}$leukemia cells in the BM of mice at 8 weeks after secondary transplantation. $\mathbf{F}$ Percentage of GFP ${ }^{+} \mathrm{CD} 25^{+}$cells in GFP ${ }^{+}$leukemia cells in the BM of Phf6 KO $+J A K 3^{M 5111}$ mice and Phf6 WT $+J A K 3^{M 5111}$ mice. G The expression of TCR- $\beta$ in GFP ${ }^{+}$CD8 ${ }^{+}$cells of Phf6 KO $+J A K 3^{M 5111}$ mice and Phf6 WT $+J A K 3^{M 5111}$ mice. H, I Phf6 KO $+J A K 3^{M 511}$ mice had more LICs $(P<0.0001)$ according to the extreme limiting dilution assay $(n=10$ per dose). J LIC analysis in mice transplanted with Phf6 knockout and wild-type leukemia cells. Confidence intervals showing $1 /$ (stem cell frequency) based on $\mathbf{H}$. 
A
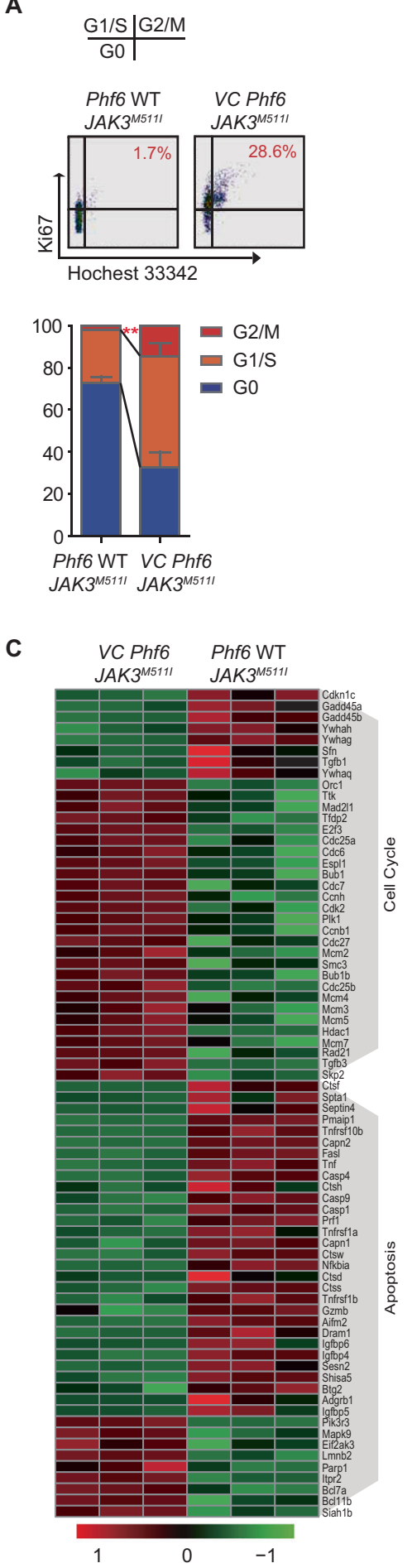

B

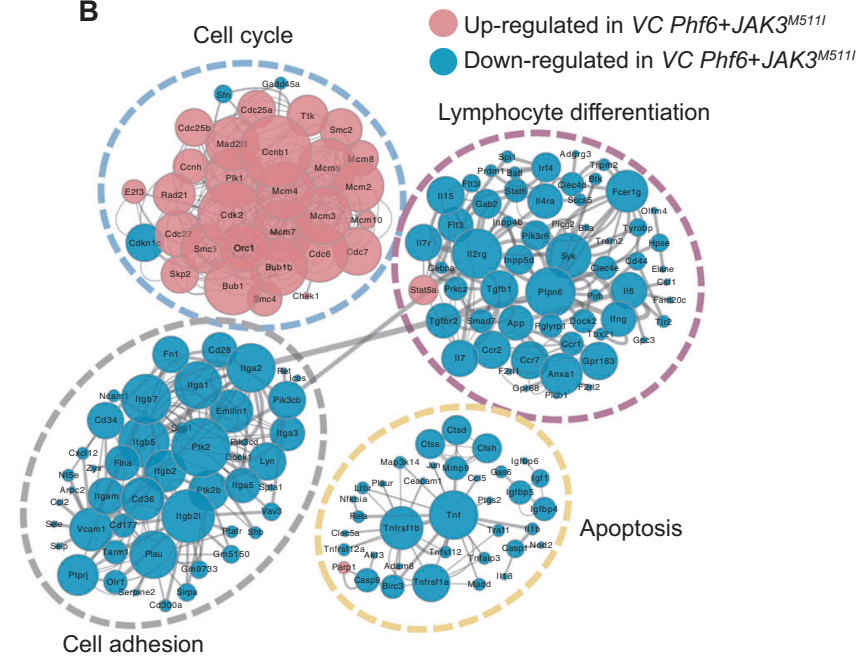

Cell cycle

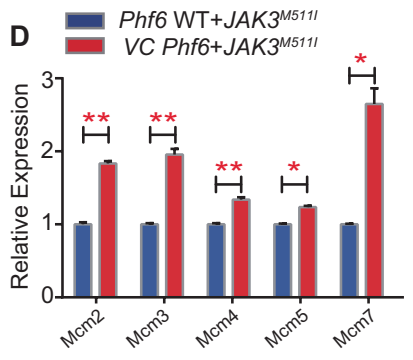

E

Apoptosis

Phf6 WT+JAK3 ${ }^{M 5111}$
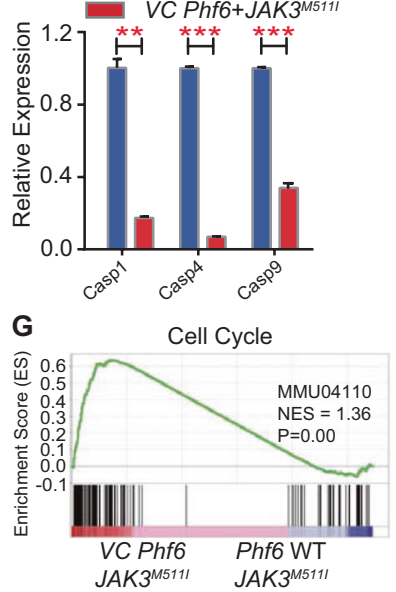

H Naive VS Effector CD8_T cell

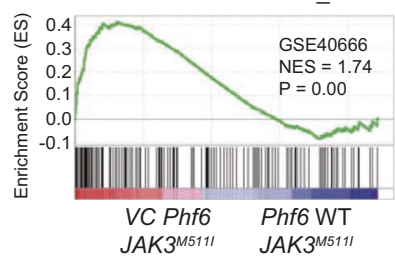

Cell cycle

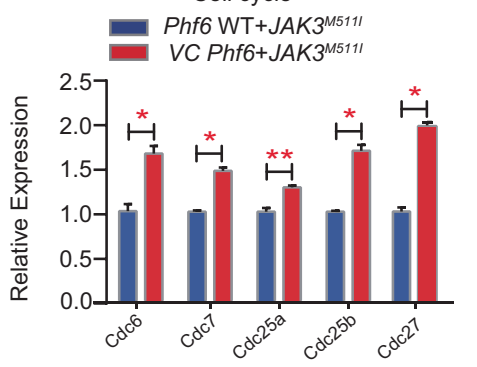

F

P53 signaling pathway Phf6 WT+JAK3 ${ }^{\text {M111 }}$

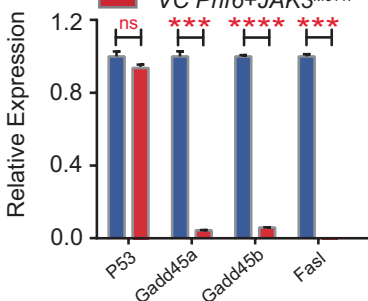

DNA Replication
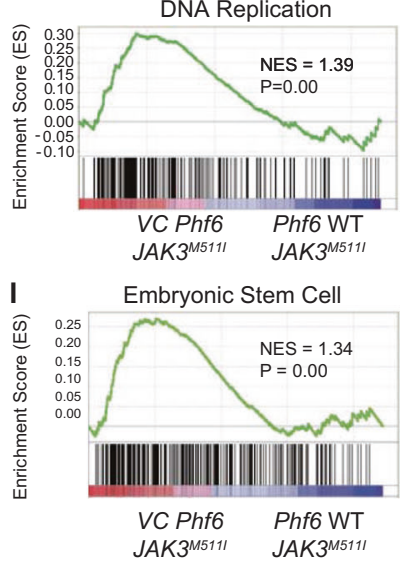

Fig. 5 Phf6 loss accelerates the JAK3 ${ }^{M 5111}$ T-ALL cell cycle transcriptional profile. A Representative FACS plots showing the cell cycle of GFP ${ }^{+}$ cells in BM (upper panel). Percentage of $\mathrm{GFP}^{+}$cells at each cell cycle stage (lower panel). B Gene interaction analysis showing the significantly altered expression pattern in genes that regulate the cell cycle, lymphocyte differentiation, cell adhesion and apoptosis in VC Phf6 + JAK3 ${ }^{M 5111}$ T-ALL cells compared with Phf6 WT + JAK3 $3^{M 5111}$ T-ALL cells. C Heatmap of cell cycle-related and apoptosis-related genes. D Validation of the expression of cell cycle-related genes in GFP ${ }^{+}$T-ALL cells. E Validation of the expression of apoptosis-related genes in GFP ${ }^{+}$T-ALL $^{-}$cells. $\mathbf{F}$ Validation of the expression of P53 signaling pathway-targeted genes in GFP ${ }^{+}$T-ALL cells. G-I Gene set enrichment analysis (GSEA) of $V C$ Phf6 + JAK3 ${ }^{\text {M5111 }}$ T-ALL cells versus Phf6 WT + JAK3 ${ }^{\text {M5111 }}$ T-ALL cells. 
A
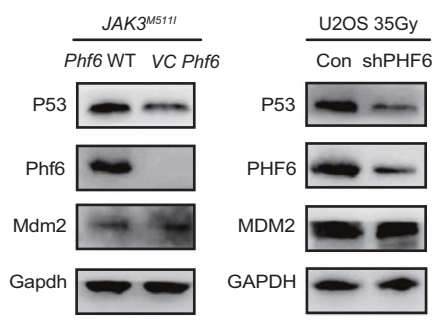

C

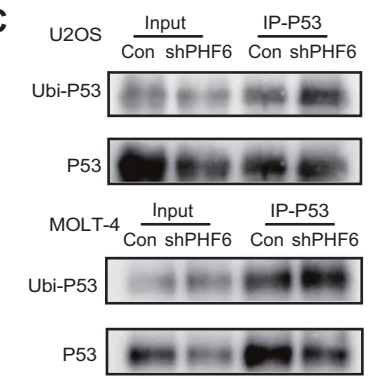

E

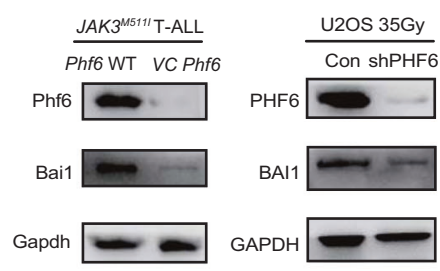

H
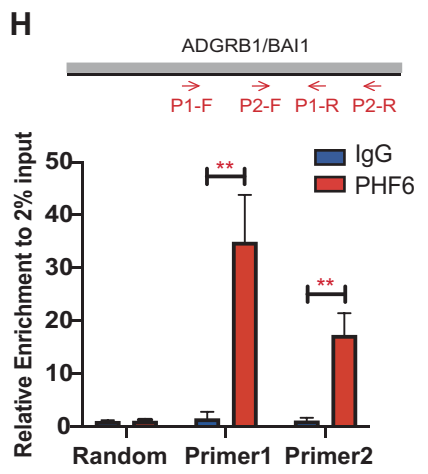

J

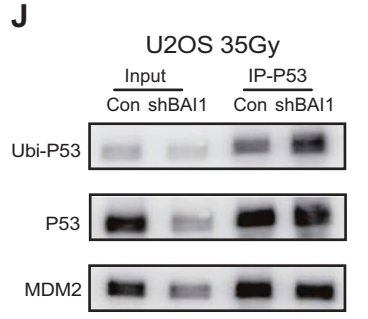

It has been reported that adhesion $G$ protein-coupled receptor B1 (ADGRB1), also known as brain-specific angiogenesis inhibitor 1 (BAI1), prevents MDM2-mediated P53 ubiquitination, and loss of BAI1 reduces P53 level [27]. We thus examined Bai1/BAl1 expression in VC Phf6 + JAK3 ${ }^{M 5111}$ cells and PHF6 KD U2OS cells,
B Phf6 WT+JAK3 $3^{M 111}$

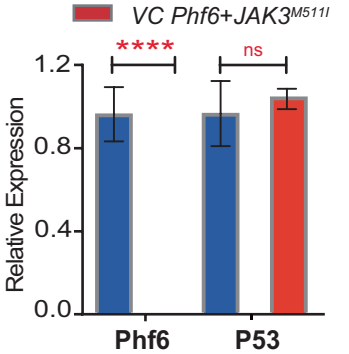

D Phf6 WT+JAK3 ${ }^{\mathrm{M} 511}$

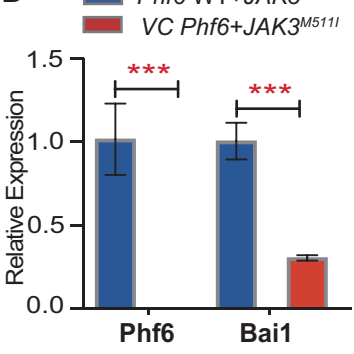

$\mathbf{F}$

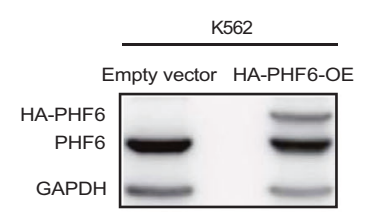

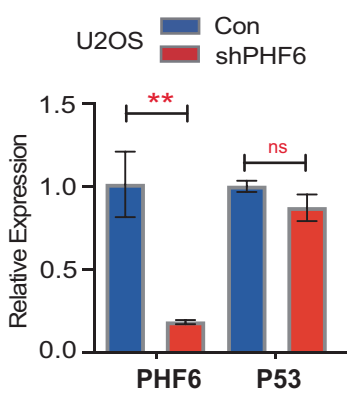

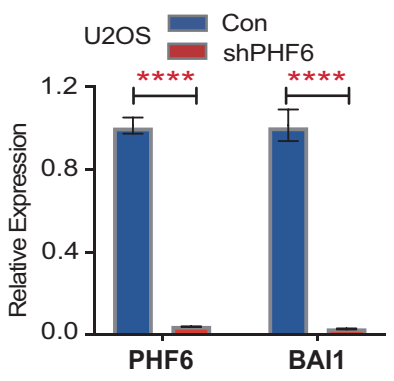

G

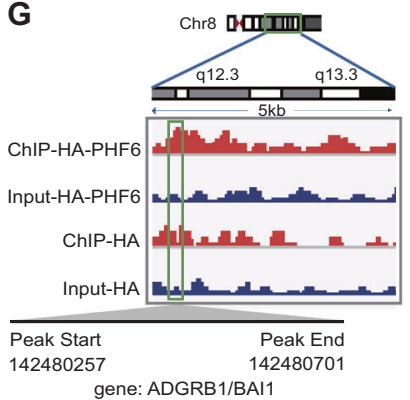

gene: ADGRB1/BAl1

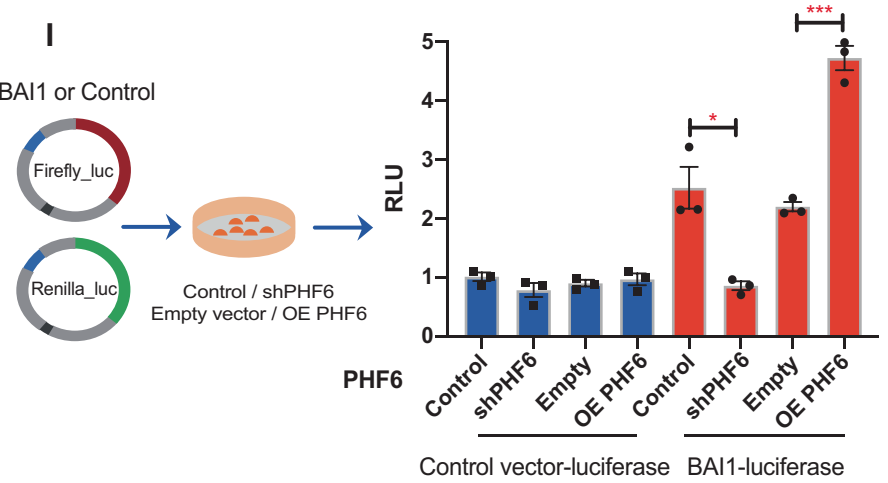

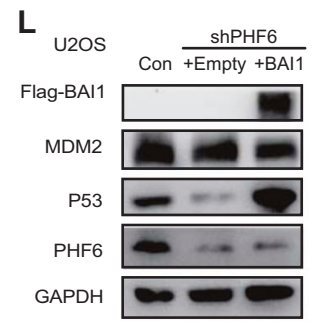

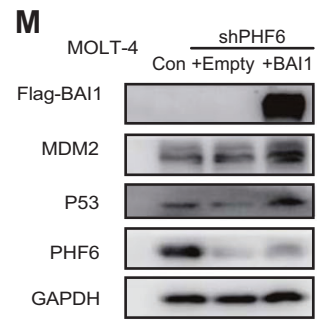

and found that the mRNA and protein expression of Bai1/BAl1 were significantly decreased in both cells in comparison with Phf6 WT $+J A K 3^{M 5111}$ or PHF6 WT U2OS cells, respectively (Fig. 6D, E). Chromatin immunoprecipitation sequencing (ChIP-seq) analysis of HA-PHF6-overexpressing (PHF6 OE) K562 cells (Fig. 6F) showed 
Fig. 6 Loss of Phf6 increases P53 degradation by decreasing Bai1 expression. A Left panel, the protein expression of P53, Mdm2 and Phf6 in Phf6 WT $+J A K 3^{M 5111}$ and VC Phf6 $+J A K 3^{M 5111}$ leukemia cells. Right panel, the protein levels of P53, MDM2, and PHF6 in PHF6 KD U2OS cells and control cells treated with X-rays ( $35 \mathrm{~Gy})$. B Left panel, the mRNA expression of P53 and Phf6 in Phf6 WT + JAK3 ${ }^{\text {M5111 }}$ and VC Phf6 + JAK3 ${ }^{\text {M5111 }}$ leukemia cells. Right panel, the mRNA expression of P53 and PHF6 in PHF6 KD U2OS cells and control cells. C Co-IP was performed with P53 antibody. P53 ubiquitination was determined by WB with anti-ubiquitin antibody in PHF6 KD/Con U2OS cells treated with X-rays (35 Gy) (upper panel) and PHF6 KD/Con MOLT-4 cells treated with $\gamma$-rays (7.5 Gy) (lower panel). D Left panel, Bai 1 and Phf6 mRNA expression in Phf6 WT $+J A K 3^{M 5111}$ and VC Phf6 + JAK3 $3^{M 111}$ leukemia cells. Right panel, BAI1 and PHF6 mRNA expression in PHF6 KD U2OS cells and control cells. E Left panel, protein levels of Phf6 and Bai 1 in Phf6 WT $+J A K 3^{M 5111}$ and VC Phf6 + JAK3 ${ }^{M 5111}$ leukemia cells. Right panel, protein levels of PHF6 and BAI1 in PHF6 KD U2OS cells and control cells treated with X-rays ( $35 \mathrm{~Gy}$ ). F The protein level of HA-PHF6 in PHF6 OE K562 cells. G PHF6 binding at the ADGRB1 (BAI1) gene locus in PHF6 OE K562 cells. H The relative amount of immunoprecipitated DNA quantified by ChIP-qPCR is given as the percentage of input DNA. I Quantification of luciferase activity from K562 cells (PHF6 KD or OE) co-transfected with a luciferase reporter containing the BAl1 sequence (or control sequence) and Renilla luciferase. J Co-IP was performed with P53 antibody. P53 ubiquitination was examined in BAI1 KD/Con U2OS cells treated with X-rays (35 Gy). $\mathbf{K}$ The protein levels of BAI1, P53, MDM2, and PHF6 in BAI1 KD/Con U2OS cells. L The protein levels of Flag-BAI1, P53, MDM2, and PHF6 in PHF6 KD + BAI1 OE U2OS cell (lane 3), PHF6 KD + Empty Vector U2OS cell (lane 2) and PHF6 Con U2OS cell (lane 1). M The protein levels of Flag-BAl1, P53, MDM2, and PHF6 in PHF6 KD + BAI1 OE MOLT-4 cell (lane 3), PHF6 KD + Empty Vector MOLT-4 cell (lane 2) and PHF6 Con MOLT-4 cell (lane 1).

that PHF6 directly bound to BAl1 gene (Fig. 6G). We used PHF6 or HA antibody to enrich PHF6 protein in PHF6-OE K562 cells and further verified the binding of PHF6 protein to the BAl1 DNA sequence by ChIP-qPCR (Figs. $6 \mathrm{H}$ and S4B). K562 cells were then co-transfected with a reporter vector containing the BAl1 sequence (or the control) inserted upstream of firefly luciferase and Renilla luciferase vector. We found that PHF6 KD reduced BAI1 luciferase activity, while PHF6 OE increased BAI1 luciferase activity (Fig. 6l). We further knocked-down BAl1 in U2OS cells and observed that BAl1 KD significantly reduced the P53 level and increased the P53 ubiquitination (Fig. 6j, k). To further investigate whether PHF6 regulates P53 expression via BAl1, the expression of BAI1 was restored in PHF6 KD U2OS cells and PHF6 KD MOLT-4 cells. We found that the expression of P53 was significantly increased in PHF6 KD U2OS cells and PHF6 KD MOLT-4 cells when BAl1 was rescued (Figs. $6 \mathrm{~L}, \mathrm{M}$ and $\mathrm{S} 4 \mathrm{C}$ ). We further investigated the interaction between PHF6 and BAI1-P53 in the Kasumi-1 cells without irradiation. We found that the mRNA and protein expressions of BAl1 were significantly decreased in PHF6 KD Kasumi-1 cells when compared with that of controls (Fig. S4D, E). The protein level of P53 was also decreased in PHF6 KD Kasumi-1 cells (Fig. S4E) and could be partially rescued when BAl1 was overexpressed in PHF6 KD Kasumi-1 cells (Fig. S4F). Taken together, our data suggested that Phf6 loss inhibited the Bai1Mdm2-P53 signaling pathway rather than by activating the Jak3/ Stat5 signaling pathway.

\section{Combined treatment with idasanutlin and tofacitinib shows greater antitumor effects in Phf6 KO $+J A K 3^{\text {M5111 }}$ T-ALL}

Since P53 signaling pathway plays a central role in the pathogenesis of Phf6 $\mathrm{KO}+J A K 3^{M 5111} \mathrm{~T}-\mathrm{ALL}$, we sought to determine whether dual activation of P53 and inactivation of

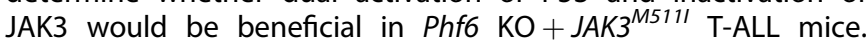
Tofacitinib is a JAK3-specific inhibitor [12]. Idasanutlin is a selective MDM2 antagonist that can activate P53 [28, 29]. VC Phf6+ $J A K 3^{M 5111}$ T-ALL mice were treated with placebo, single tofacitinib, single idasanutlin, or combined tofacitinib + idasanutlin (To + Id) (Fig. 7A). We measured p-Stat5 and P53 in VC Phf6 + JAK3 ${ }^{\text {M5111 }} \mathrm{BM}$ cells from treated or nontreated mice. We found that $p$-Stat 5 was decreased (Figs. 7B and S5A), and P53 was increased in mice treated with To + Id (Fig. 7C). The survival time of mice treated with To + Id was significantly longer than mice treated with placebo, single tofacitinib or idasanutlin (Fig. 7D). The counts of hemoglobin and PLT were increased, while the count of leukemia cells was decreased in the PB of mice treated with To + Id when compared with mice in other groups (Fig. 7E). Additionally, the percentages of $\mathrm{GFP}^{+}$leukemia cells in the PB, BM, spleen, liver, and thymus were decreased in mice treated with To + Id when compared with other groups (Figs. 7F and S5B-E). The weights of spleen, liver, and thymus were significantly decreased in mice treated with To + Id in comparison with other groups (Fig. S5F, G). $\mathrm{HE}$ staining showed that the degree of leukemia cell infiltration in the spleen, liver, brain, thymus, and BM was reduced in mice treated with To + Id when compared with other groups (Fig. 7G). Immunohistochemical staining of Ki67, a marker of cell proliferation, showed significantly reduced staining in the To + Id-treated group when compared with the other groups (Fig. $\mathrm{S} 5 \mathrm{H}$ ), while TUNEL (TdT-mediated dUTP nick-end labeling) staining showed a significant increase in apoptotic cells in mice treated with To + Id than other groups (Fig. 7G).

Also, we constructed JAK3 ${ }^{M 5111}$-overexpression MOLT-4 cells that were treated with shPHF6 (PHF6 KD) or sh-Control (PHF6 Con464) respectively, and confirmed by WB along with MOLT-4 cells (WT) (Fig. $7 \mathrm{H})$. We found that the proliferation of PHF6 KD $+J A K 3^{M 5111}$ cells was much faster than PHF6 Con464 JAK3 $3^{M 111}$ or other control cells (Fig. 7l). Furthermore, the proliferation of PHF6 KD + JAK3 ${ }^{\text {M5111 }}$ MOLT-4 cells was significantly decreased while their percentage of apoptosis was increased after treated with To + Id when compared with single drug or control treatment groups (Fig. 7J, K). Our result thus demonstrated that combination therapy with tofacitinib and idasanutlin can reduce leukemia burden better than single drug treatment in Phf6 KO $+J A K 3^{M 5111}$ mice, which was also validated by the result in human MOLT4 cells.

\section{DISCUSSION}

Understanding the mechanism of how epigenetic regulatory genes target chromatin and redirect gene transcription and activation in leukemogenesis is imperative for developing novel therapies. As a chromatin remodeling-related gene, PHF6 is frequently mutated in T-ALL patients $[13,17,30]$. Here, we demonstrated that PHF6 mutations more commonly coexisted with JAK/STAT mutations in T-ALL patients (Fig. 1A). The average survival time of patients with JAK/STAT and PHF6 comutation was shorter than that of patients without this comutation (Figs. 1E and S1A). Phf6 deletion led to rapid development of JAK3 ${ }^{M 5111}$-induced T-ALL by inhibiting the Mdm2-P53 signaling pathway. Leukemia progression can be contained better by specific inhibition of JAK3 and Mdm2-P53 in Phf6-deficient and JAK3 ${ }^{M 5111}$ T-ALL cells (Fig. 7D). Furthermore, we revealed that the progression of JAK3 $3^{M 111}$ induced T-ALL from low to high malignancy is triggered by the coexistence of PHF6 mutation, and that provided a potential therapeutic window for the modulation of P53 and JAK3 activity in the treatment of T-ALL patients with PHF6 and JAK3 comutation.

It has been reported that Phf6 is essential for HSC homeostasis and T-ALL initiation, although Phf6 appears to play a modest role in normal T-cell differentiation and proliferation [20]. Loss of Phf6 slightly reduced number of T cells in PB and BM, but did not lead to spontaneous hematological malignant transformation in mice 
A

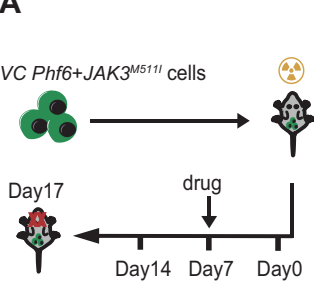

E

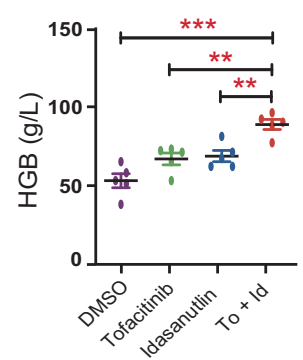

B
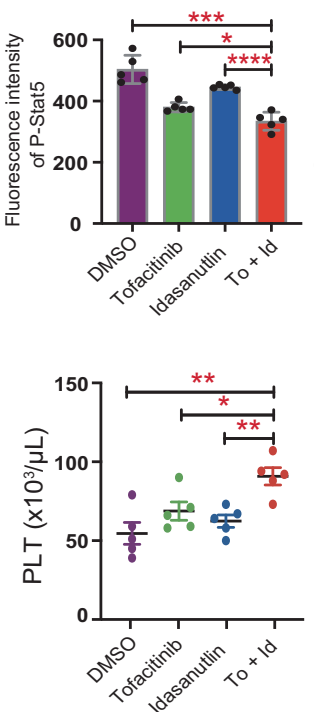

C

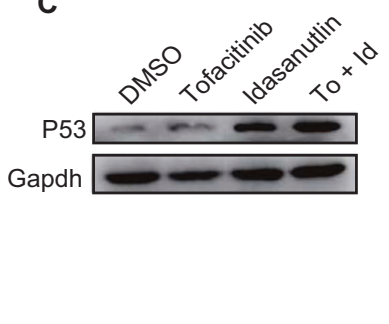

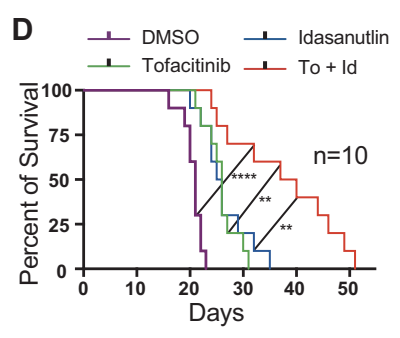

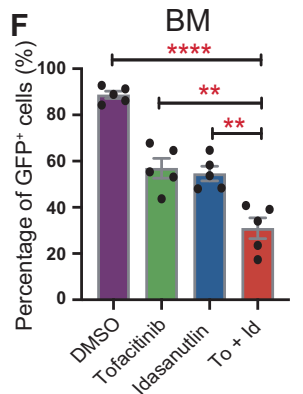

G

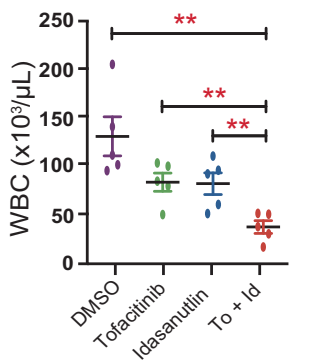

\begin{abstract}
HE
\end{abstract}

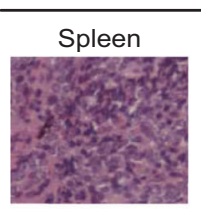
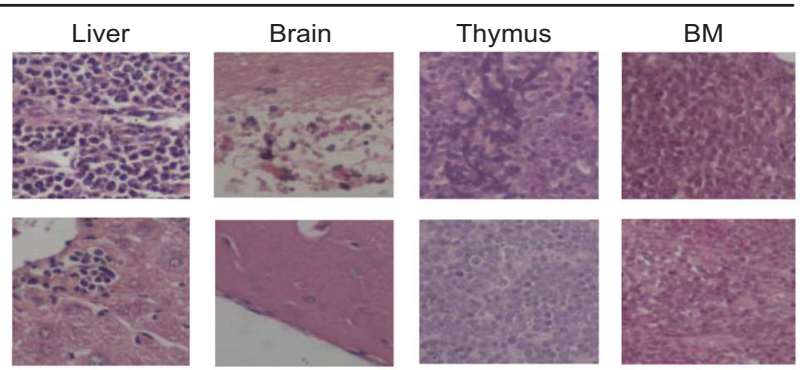

TUNEL
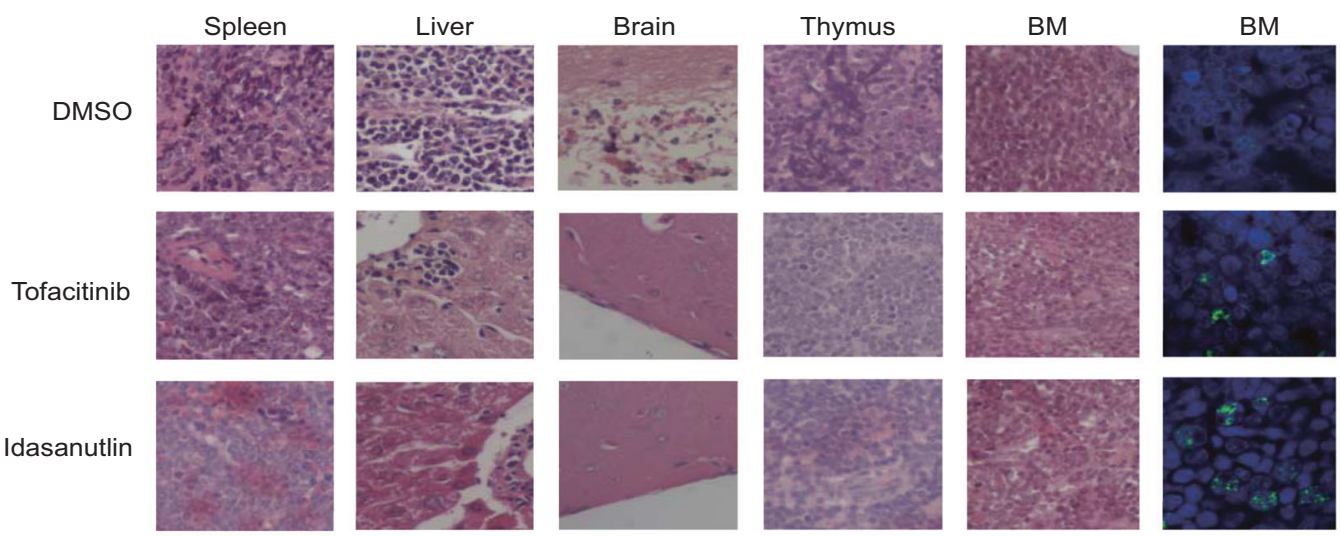

Idasanutlin
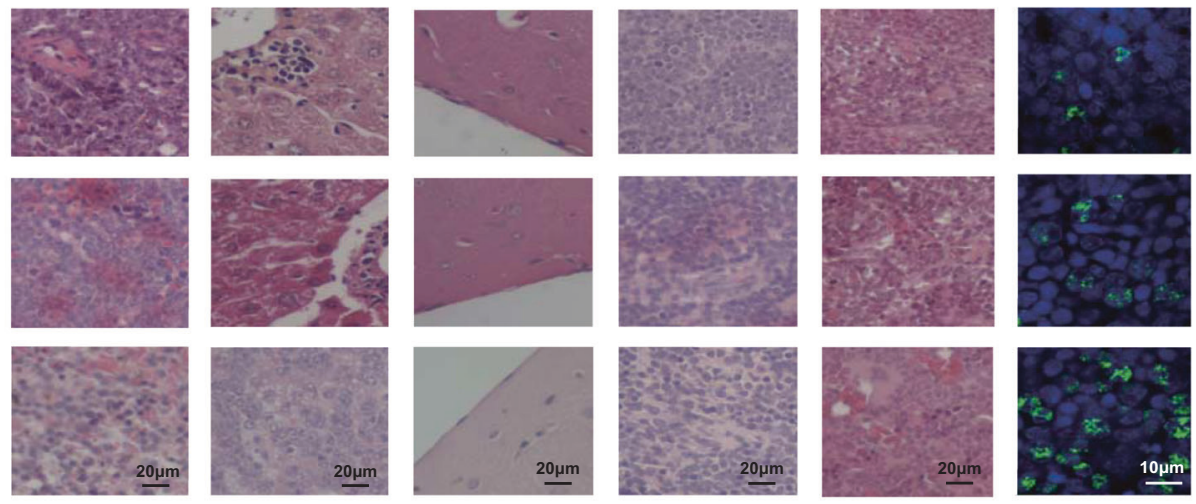

To + Id
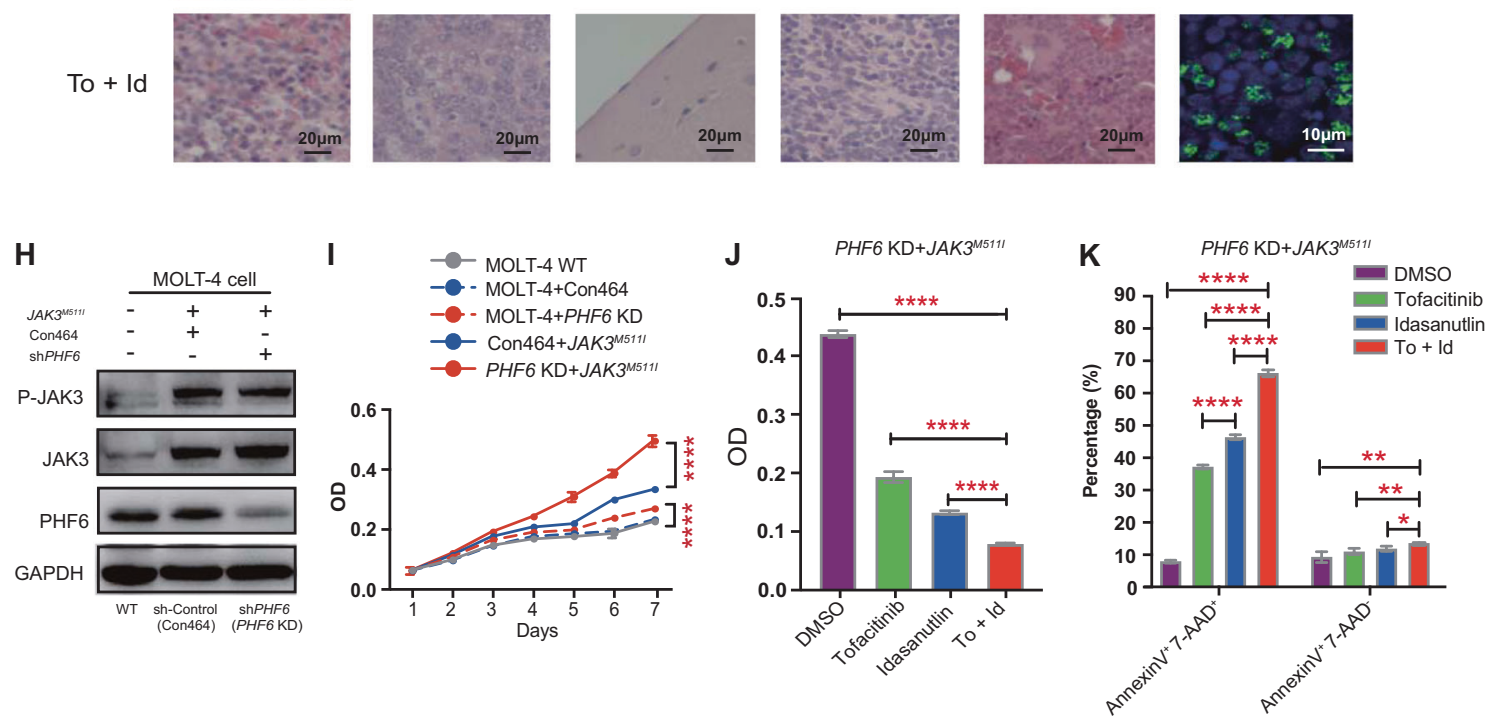

[20]. Earlier studies theorized that PHF6 may play important roles in lineage-specification during leukemogenesis. For example, lack of Phf6 promoted Notch-induced T-ALL initiation and MLL-AF9induced $A M L$ progression, while decelerated the development of BCR-ABL1-induced B-ALL [31]. However, Thomas and colleagues

suggested that PHF6 might not act in a strictly lineage-dependent manner. They found that Phf6 knockout accelerated TLX1/TLX3induced B-ALL [22], and that is different from the oncogenic role of Phf6 in BCR-ABL1-induced B-ALL. It indicated that the role(s) of PHF6 may depend on the combination of oncogenic mutations 


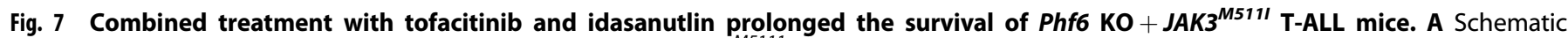
representation of different drug treatments in VC Phf6 $+J A K 3^{M 5111}$ T-ALL mice. B Phosphoflow cytometry was used to measure the phosphorylation level of Stat 5 in leukemia cells from VC Phf6 + JAK3 ${ }^{M 5111}$ T-ALL mice treated with placebo, single tofacitinib, single idasanutlin, or combined tofacitinib and idasanutlin through oral gavage. C Western blotting was used to assess the expression of P53 in leukemia cells from VC Phf6 $+J A K 3^{M 5111}$ T-ALL mice treated with different drugs. D Kaplan-Meier survival curves of VC Phf6 + JAK3 ${ }^{M 5111}$ T-ALL mice treated with different drugs ( $n=10$ per group). Mice treated with combined tofacitinib and idasanutlin VS placebo (log-rank test $P<0.0001)$, single tofacitinib (log-rank test $P=0.0019$ ) or single idasanutlin (log-rank test $P=0.0034$ ). E The counts of HGB, PLT, and WBC in PB by routine blood tests. F The percentage of $\mathrm{GFP}^{+}$leukemia cells in BM from VC Phf6 $+J A K 3^{M 5111} \mathrm{~T}$-ALL mice treated with different drugs. G Immunohistochemical staining for hematoxylin and eosin (HE) (magnification, $\times 40$ ) and TUNEL staining (green represents TUNEL, blue represents DAPI, magnification, $\times 60$ ) in $\mathrm{BM}$, spleen, liver, brain, and thymus from VC Phf6 $+J A K 3^{M 5111}$ T-ALL mice treated with different drugs. H Construction of PHF6 KD + JAK3 ${ }^{M 5111}$ and PHF6 Con464 $+J A K 3^{M 5111}$ MOLT-4 cells and their WB verification. I The proliferation of MOLT-4 PHF6 $\mathrm{KD}+J_{A K 3^{M 5111}}$ cells was much faster than MOLT-4 PHF6 Con464 + JAK3 ${ }^{M 5111}$, MOLT-4 PHF6 KD, MOLT-4 Con464 or MOLT-4 WT cells respectively. J, K Proliferation and apoptosis of MOLT-4 PHF6 KD + JAK3 ${ }^{M 5111}$ cells were examined in vitro with various drug treatment of $0.2 \%$ DMSO, single tofacitinib $(2.0 \mu \mathrm{M})$, single idasanutlin $(0.5 \mu \mathrm{M})$ or combined tofacitinib + idasanutlin for $48 \mathrm{~h}$.

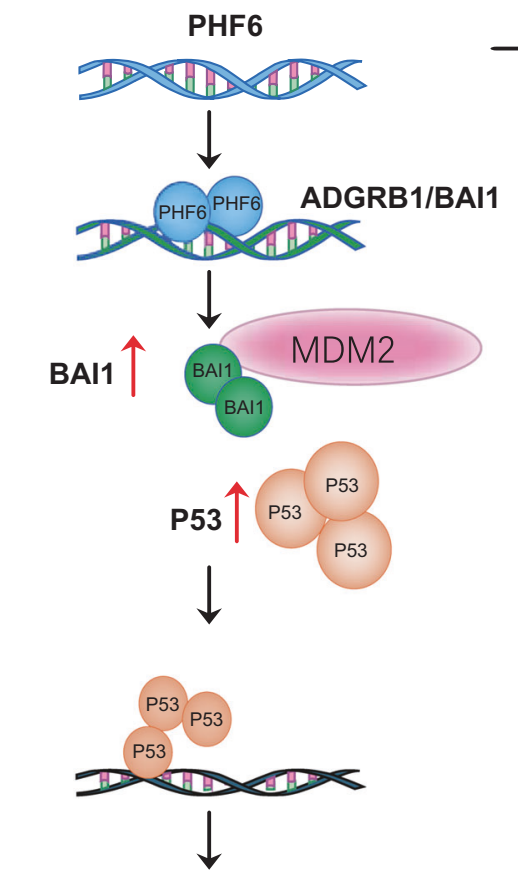

Suppressing leukemia

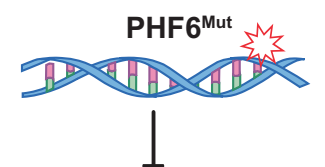

ADGRB1/BAI1

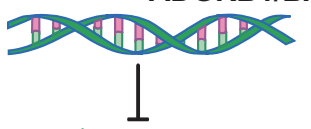

BAl1 $\downarrow$
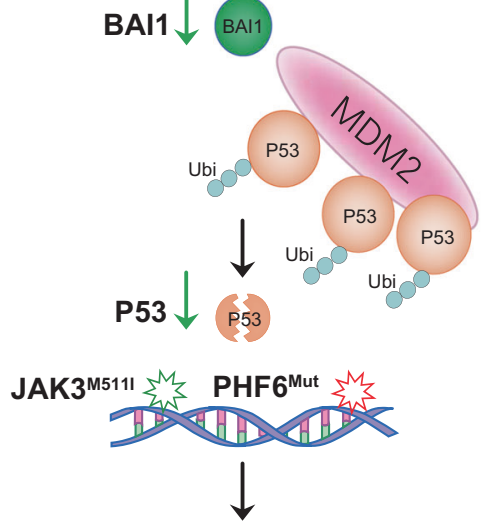

Promoting leukemia

Fig. 8 A proposed model for the role of PHF6 in T-ALL initiation by regulating BAI1-MDM2-P53 signaling pathway. In JAK3 ${ }^{M 5111}$-induced leukemia, PHF6 could bind to ADGRB1 (BA/1) gene, increase its expression, upregulate BAl1 level, prevent MDM2-mediated P53 ubiquitination, stabilize the P53 protein, and suppress leukemia development (Left panel). In the same leukemia model, loss of PHF6 could downregulate the expression of BAI1, lead to increased MDM2-P53 binding and P53 degradation, and accelerate JAK3 ${ }^{M 5111}$-induced T-ALL progression (Right panel).

and specific molecular pathways that drive leukemia. In our studies, we found that primary Phf6 KO $+J A K 3^{M 5111}$ progenitor cells developed a complex hematolymphoid neoplasm characterized by the coexistence of different populations of atypical cells (Fig. 2l, J). However, when we depleted Phf6 in MC Phf6+ $J A K 3^{M 5111}$ mice with plpC, leukemia cells were mainly lymphoid cells (Fig. 3D, E). These results suggested that if PHF6 mutation and JAK3 mutation occurred in hematopoietic progenitors, it may induce a complex hematolymphoid neoplasm. When PHF6 mutation acted as "a secondary strike" in JAK3 $3^{M 111}$-induced TALL, it might also promote the T-ALL progression. Based on our observations, we reasoned that PHF6 might function as a tumor suppressor, while PHF6 loss or mutations favors T-ALL initiation by lowering the threshold for subsequent oncogenic transformation in hematopoietic progenitors, and postulated the role in promoting T-ALL development.

We further demonstrated how PHF6 functions in $J A K 3^{M 511 I_{-}}$ induced T-ALL independent of the Jak3-Stat5 signaling pathway. As expected, we found that Stat5 was activated in both Phf6 KO/ WT $+J A K 3^{M 5111}$ cells (Fig. S4A) [12]. However, the Mdm2-P53 signaling pathway was inhibited only in Phf6 KO $+J A K 3^{M 5111}$ but not in Phf6 WT $+J A K 3^{M 5111}$ leukemia cells (Fig. 6A). This suggested that T-ALL progression due to Phf6 loss is dependent on additional signaling pathways. BAI1 belongs to the adhesion subgroup of GPCRs, which functions at preventing MDM2mediated P53 ubiquitination, and its loss could substantially reduce the P53 level [27]. Consistent with prior findings showing that P53 loss increased penetrance and accelerated progression of leukemia [28, 32], we showed here that PHF6 loss increased $J A K 3^{M 5111}$-induced T-ALL initiation by downregulating BAI1 expression, thereby decreasing BAl1 and MDM2 interaction, and destabilizing P53 (modeled in Fig. 8). Thus, our results defined a PHF6-BAI1-P53 signaling axis and linked it with JAK3 $3^{M 5111}$-induced T-ALL genesis. It will be interesting to assess whether T-ALL patients with JAK3 and PHF6 comutation could further benefit from combined therapy with MDM2 and JAK3 inhibitors.

In conclusion, we revealed that PHF6 and JAK3 mutations cooperatively drive T-ALL progression probably via inhibiting the BAI1-MDM2-P53 signaling pathway, in addition to activating the JAK3/STAT5 signaling pathway. We further demonstrated that 
combination therapy with tofacitinib and idasanutlin reduced the Phf6 KO $+J A K 3^{M 5111}$ leukemia burden in vivo. Our study suggested that the combined usage of JAK3 and MDM2 inhibitors should increase the drug benefit for T-ALL patients with PHF6 and JAK3 comutation.

\section{REFERENCES}

1. Belver $L$, Ferrando $A$. The genetics and mechanisms of $T$ cell acute lymphoblastic leukaemia. Nat Rev Cancer. 2016;16:494-507.

2. Juliusson G, Hough R. Leukemia. Prog Tumor Res. 2016;43:87-100.

3. Marks DI, Rowntree C. Management of adults with T-cell lymphoblastic leukemia. Blood 2017;129:1134-42.

4. Albertí-Servera L, Demeyer S, Govaerts I, Swings T, Cools J. Single-cell DNA amplicon sequencing reveals clonal heterogeneity and evolution in T-cell acute lymphoblastic leukemia. Blood 2020;137:801-11.

5. Van Vlierberghe $P$, Ferrando $A$. The molecular basis of $T$ cell acute lymphoblastic leukemia. J Clin Investig. 2012;122:3398-406.

6. Yeh T-C, Liang D-C, Liu H-C, Jaing $\mathrm{T}-\mathrm{H}$, Chen $\mathrm{S}-\mathrm{H}$, Hou J-Y, et al. Clinical and biological relevance of genetic alterations in pediatric T-cell acute lymphoblastic leukemia in Taiwan. Pediatr Blood Cancer. 2019;66:e27496.

7. Dorritie KA, McCubrey JA, Johnson DE. STAT transcription factors in hematopoiesis and leukemogenesis: opportunities for therapeutic intervention. Leukemia 2014;28:248-57.

8. de Bock CE, Demeyer S, Degryse S, Verbeke D, Sweron B, Gielen O, et al. HOXA9 Cooperates with Activated JAK/STAT Signaling to Drive Leukemia Development. Cancer Discov. 2018;8:616-31.

9. Xiao W, Bharadwaj M, Levine M, Farnhoud N, Pastore F, Getta BM, et al. PHF6 and DNMT3A mutations are enriched in distinct subgroups of mixed phenotype acute leukemia with T-lineage differentiation. Blood Adv. 2018;2:3526-39.

10. Flex E, Petrangeli V, Stella L, Chiaretti S, Hornakova T, Knoops L, et al. Somatically acquired JAK1 mutations in adult acute lymphoblastic leukemia. J Exp Med. 2008;205:751-8.

11. Vainchenker W, Constantinescu SN. JAK/STAT signaling in hematological malignancies. Oncogene. 2013;32:2601-13.

12. Degryse S, de Bock CE, Cox L, Demeyer S, Gielen O, Mentens N, et al. JAK3 mutants transform hematopoietic cells through JAK1 activation, causing T-cell acute lymphoblastic leukemia in a mouse model. Blood. 2014;124:3092-100.

13. Vicente C, Schwab C, Broux M, Geerdens E, Degryse S, Demeyer S, et al. Targeted sequencing identifies associations between IL7R-JAK mutations and epigenetic modulators in T-cell acute lymphoblastic leukemia. Haematologica. 2015;100:1301-10.

14. Asnafi V, Noir SL, Lhermitte L, Gardin C, Legrand F, Vallantin X, et al. JAK1 mutations are not frequent events in adult T-ALL: a GRAALL study. $\mathrm{Br} J$ Haematol. 2010;148:178-79.

15. Lin PH, Li HY, Fan SC, Yuan TH, Chen M, Hsu YH, et al. A targeted next-generation sequencing in the molecular risk stratification of adult acute myeloid leukemia: implications for clinical practice. Cancer Med. 2017;6:349-60.

16. Tashi T, Swierczek S, Prchal JT. Familial MPN predisposition. Curr Hematol Malig Rep. 2017;12:442-47.

17. Liu Y, Easton J, Shao Y, Maciaszek J, Wang Z, Wilkinson MR, et al. The genomic landscape of pediatric and young adult T-lineage acute lymphoblastic leukemia. Nat Genet. 2017;49:1211-18.

18. Zhou X, Gu Y, Han Q, Soliman M, Song C, Ge Z. Coexistence of EZH2, NOTCH1, IL7R, and PHF6 mutations in adult T-cell acute lymphoblastic leukemia. Turkish J Haematol. 2017;34:366-68.

19. Alexander TB, Gu Z, lacobucci I, Dickerson K, Choi JK, Xu B, et al. The genetic basis and cell of origin of mixed phenotype acute leukaemia. Nature. 2018;562:373-79.

20. Hsu YC, Chen TC, Lin CC, Yuan CT, Hsu CL, Hou HA, et al. Phf6-null hematopoietic stem cells have enhanced self-renewal capacity and oncogenic potentials. Blood Adv. 2019;3:2355-67.

21. Wendorff AA, Quinn SA, Rashkovan M, Madubata CJ, Ambesi-Impiombato A, Litzow MR, et al. Phf6 loss enhances HSC self-renewal driving tumor initiation and leukemia stem cell activity in T-ALL. Cancer Discov. 2019;9:436-51.

22. McRae HM, Garnham AL, Hu Y, Witkowski MT, Corbett MA, Dixon MP, et al. PHF6 regulates hematopoietic stem and progenitor cells and its loss synergizes with expression of TLX3 to cause leukemia. Blood. 2019;133:1729-41.

23. Miyagi S, Sroczynska P, Kato Y, Nakajima-Takagi Y, Oshima M, Rizq O, et al. The chromatin-binding protein Phf6 restricts the self-renewal of hematopoietic stem cells. Blood. 2019;133:2495-506.

24. Hu Y, Smyth GK. ELDA: extreme limiting dilution analysis for comparing depleted and enriched populations in stem cell and other assays. J Immunol Methods. 2009;347:70-8.
25. Spinella JF, Cassart P, Richer C, Saillour V, Ouimet M, Langlois S, et al. Genomic characterization of pediatric T-cell acute lymphoblastic leukemia reveals novel recurrent driver mutations. Oncotarget. 2016;7:65485-503.

26. Habu S, Okumura K, Diamantstein T, Shevach EM. Expression of interleukin 2 receptor on murine fetal thymocytes. Eur J Immunol. 1985;15:456-60.

27. Dan Z, Satoru O, Zhaobin Z, Reichert ZR, Liquan $Y$, Yonehiro $K$, et al. BAl1 suppresses medulloblastoma formation by protecting p53 from Mdm2mediated degradation. Cancer Cell. 2018;33:1004-16.

28. Ciardullo C, Aptullahoglu E, Woodhouse L, Lin WY, Wallis JP, Marr H, et al. Nongenotoxic MDM2 inhibition selectively induces a pro-apoptotic p53 gene signature in chronic lymphocytic leukemia cells. Haematologica. 2019;104:2429-42.

29. Lehmann C, Friess T, Birzele F, Kiialainen A, Dangl M. Superior anti-tumor activity of the MDM2 antagonist idasanutlin and the Bcl-2 inhibitor venetoclax in p53 wild-type acute myeloid leukemia models. J Hematol Oncol. 2016;9:50.

30. Van Vlierberghe $\mathrm{P}$, Palomero $\mathrm{T}$, Khiabanian $\mathrm{H}$, Van der Meulen J, Castillo $\mathrm{M}$, Van Roy N, et al. PHF6 mutations in T-cell acute lymphoblastic leukemia. Nat Genet. 2010;42:338-42.

31. Soto-Feliciano YM, Bartlebaugh JME, Liu Y, Sanchez-Rivera FJ, Bhutkar A, Weintraub AS, et al. PHF6 regulates phenotypic plasticity through chromatin organization within lineage-specific genes. Genes Dev. 2017;31:973-89.

32. Zhang L, McGraw KL, Sallman DA, List AF. The role of p53 in myelodysplastic syndromes and acute myeloid leukemia: molecular aspects and clinical implications. Leuk Lymphoma. 2017;58:1777-90.

\section{ACKNOWLEDGEMENTS}

This work was supported by funds from the Ministry of Science and Technology of China (2018YFA0107801 and 2017YFA0103402 to WPY); the Chinese Academy of Medical Sciences Innovation Fund for Medical Sciences, CIFMS (2017-12M-3-015 to WPY and XMW and 2016-12M-1-017 to TC and YJC); and the National Natural Science Foundation of China (81629001 to WPY, 82070169 and 81870088 to XMW, 81970149 and 81770105 to WPY).

\section{AUTHOR CONTRIBUTIONS}

WPY and XMW conceived the project, supervised the research and revised the paper. SNY, SBH and XMW performed most of the experiments and analyzed the data. TXG, SY, YJL, FZ, JG and YXW assisted with the mouse experiments, flow cytometry analysis and data processing. XMW and SNY wrote the manuscript. YJC, JS and TC contributed to the data analyses and paper discussion. All authors read and approved the final manuscript.

\section{COMPETING INTERESTS}

The authors declare no competing interests.

\section{ADDITIONAL INFORMATION}

Supplementary information The online version contains supplementary material available at https://doi.org/10.1038/s41375-021-01392-1.

Correspondence and requests for materials should be addressed to X.W. or W.Y.

Reprints and permission information is available at http://www.nature.com/ reprints

Publisher's note Springer Nature remains neutral with regard to jurisdictional claims in published maps and institutional affiliations.

Open Access This article is licensed under a Creative Commons Attribution 4.0 International License, which permits use, sharing, adaptation, distribution and reproduction in any medium or format, as long as you give appropriate credit to the original author(s) and the source, provide a link to the Creative Commons license, and indicate if changes were made. The images or other third party material in this article are included in the article's Creative Commons license, unless indicated otherwise in a credit line to the material. If material is not included in the article's Creative Commons license and your intended use is not permitted by statutory regulation or exceeds the permitted use, you will need to obtain permission directly from the copyright holder. To view a copy of this license, visit http://creativecommons. org/licenses/by/4.0/.

(c) The Author(s) 2021 